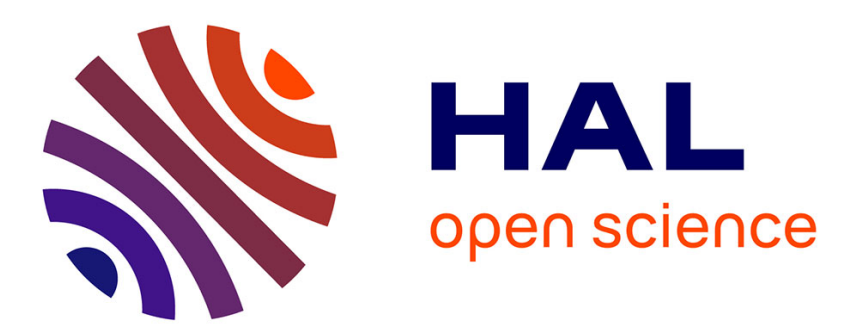

\title{
Strain localization analysis using a large deformation anisotropic elastic-plastic model coupled with damage
}

Badis Haddag, Farid Abed-Meraim, Tudor Balan

\section{To cite this version:}

Badis Haddag, Farid Abed-Meraim, Tudor Balan. Strain localization analysis using a large deformation anisotropic elastic-plastic model coupled with damage. International Journal of Plasticity, 2008, pp.1970-1996. 10.1016/j.ijplas.2008.12.013 . hal-01206515

\section{HAL Id: hal-01206515 \\ https://hal.science/hal-01206515}

Submitted on 29 Sep 2015

HAL is a multi-disciplinary open access archive for the deposit and dissemination of scientific research documents, whether they are published or not. The documents may come from teaching and research institutions in France or abroad, or from public or private research centers.
L'archive ouverte pluridisciplinaire HAL, est destinée au dépôt et à la diffusion de documents scientifiques de niveau recherche, publiés ou non, émanant des établissements d'enseignement et de recherche français ou étrangers, des laboratoires publics ou privés. 


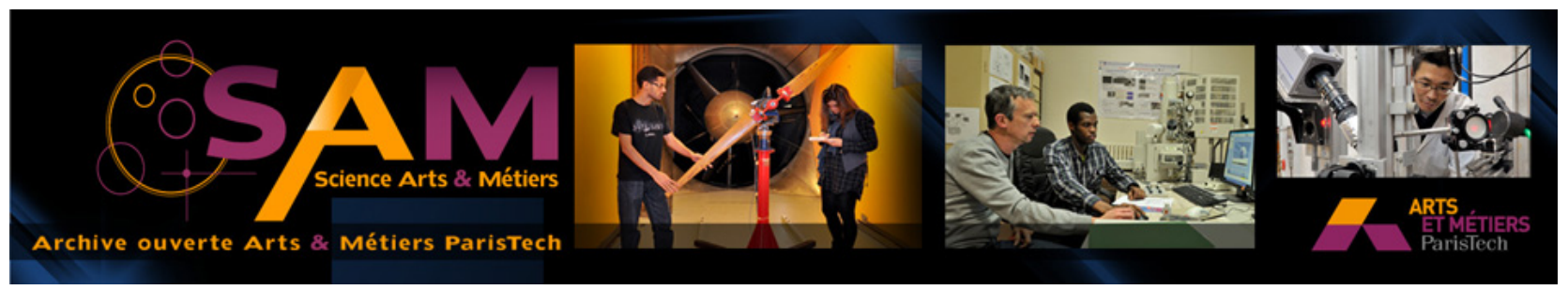

\section{Science Arts \& Métiers (SAM)}

is an open access repository that collects the work of Arts et Métiers ParisTech researchers and makes it freely available over the web where possible.

This is an author-deposited version published in: http://sam.ensam.eu

Handle ID: .http://hdl.handle.net/10985/10212

\section{To cite this version :}

Badis HADDAG, Farid ABED-MERAIM, Tudor BALAN - Strain localization analysis using a large deformation anisotropic elastic-plastic model coupled with damage - International Journal of Plasticity p.1970-1996 - 2008 


\title{
Strain localization analysis using a large deformation anisotropic elastic-plastic model coupled with damage
}

\author{
Badis Haddag, Farid Abed-Meraim* and Tudor Balan
}

Laboratoire de Physique et Mécanique des Matériaux, LPMM, UMR CNRS 7554, ENSAM de Metz, Arts et Métiers ParisTech, 4 rue Augustin Fresnel, 57078 Metz Cedex 3, France

Keywords: Strain Localization, Anisotropic Elastic-plasticity, Large Strain, IsotropicKinematic Hardening, Continuum Damage Theory, Shear Band, Finite Element Simulation.

* Corresponding author. Address: ENSAM de Metz, 4 rue Augustin Fresnel, 57078 Metz

Cedex 03, France. Tel.: +(33) 3.87.37.54.79; Fax: +(33) 3.87.37.54.70.

E-mail address: farid.abed-meraim@metz.ensam.fr 


\begin{abstract}
Sheet metal forming processes generally involve large deformations together with complex loading sequences. In order to improve numerical simulation predictions of sheet parts forming, physically-based constitutive models are often required. The main objective of this paper is to analyze the strain localization phenomenon during the plastic deformation of sheet metals in the context of such advanced constitutive models. Most often, an accurate prediction of localization requires damage to be considered in the finite element simulation. For this purpose, an advanced, anisotropic elastic-plastic model, formulated within the large strain framework and taking strain-path changes into account, has been coupled with an isotropic damage model. This coupling is carried out within the framework of continuum damage mechanics. In order to detect the strain localization during sheet metal forming, Rice's localization criterion has been considered, thus predicting the limit strains at the occurrence of shear bands as well as their orientation. The coupled elastic-plastic-damage model has been implemented in Abaqus/Implicit. The application of the model to the prediction of Forming Limit Diagrams (FLDs) provided results that are consistent with the literature and emphasized the impact of the hardening model on the strain-path dependency of the FLD. The fully threedimensional formulation adopted in the numerical development allowed for some new results - e.g. the out-of-plane orientation of the normal to the localization band, as well as more realistic values for its in-plane orientation.
\end{abstract}




\section{Introduction}

The numerical prediction of metal forming defects is a constant preoccupation both for scientists and industry. With the development of new grades of sheet metals with high performances (i.e. combining large ductility with high strength), the study of their formability limits has become of great importance, since it contributes to increasing the design efficiency by reducing costs and time. In order to characterize the formability of sheet metals, Keeler (1965) introduced the concept of Forming Limit Diagram, which is defined by the two values of the principal strains in the sheet plane. This diagram represents a real practical interest, in the sense that it defines a safe zone corresponding to the domain of the sheet formability. Since this early work of Keeler, several efforts have been devoted to the prediction of such diagrams (e.g. Laukonis and Gosh, 1978; Hutchinson and Neale, 1978; Cordebois and Ladevèze, 1982; Needleman and Tvergaard, 1983; Brunet et al., 1985; Arrieux et al., 1985; Kuroda and Tvergaard, 2000a,b; Stoughton, 2000; Chow et al., 2002, Butuc et al., 2006).

The use of advanced constitutive models is known to contribute to the proper prediction of the stress/strain history of the material under complex loading and, thus, to an improved prediction of the formability limit. The accurate description of the material behavior has received considerable attention in the last decades. For simple applications, the Swift and Voce laws are widely used to reproduce the isotropic hardening that occurs during monotonic loading paths. However, strain-path changes induce more complex phenomena that must be considered in the constitutive model. Among the phenomenological approaches, more advanced models are defined by combining isotropic and kinematic hardening. In these models, several internal variables with nonlinear evolution laws are introduced in order to improve the description of the Bauschinger effect, the ratcheting effect in fatigue, etc. (e.g. Marquis, 1979;

Chaboche, 1986; Lemaitre and Chaboche, 1985). An extensive bibliography on this subject is reported in (Haddag et al., 2007) as well as in (Chaboche, 2008).

Since hardening in sheet metals is essentially due to the dislocation microstructure and its evolution, numerous attempts have been made to describe its effect on hardening at a macroscopic scale. Following this approach, Teodosiu and $\mathrm{Hu}(1995,1998)$ and Teodosiu (1997) proposed a microstructure-based model representing not only the monotonic or reverse loading, but also the whole range between the two, including the particular case of an orthogonal strain-path. More precisely, the introduction of physically-motivated internal variables that describe the evolution of the persistent dislocation structures allowed new transient phenom- 
ena to be modeled. Stagnation, softening, and rapid changes in the work hardening rate - as observed during abrupt, two-stage sequential rheological tests - for a wide range of sheet metals are very well described with this model (Haddadi et al., 2006). In the current work, the plastic anisotropy induced by hardening has been modeled with the Teodosiu and $\mathrm{Hu}$ (1998) hardening model. The model can be coupled with any yield potential to take into account initial anisotropy. The classical cyclic hardening model of Chaboche, combining the ArmstrongFrederick and Voce (or Swift) laws, is used as a reference model, since it is widely available in commercial finite element codes.

However, sheet metal forming involves large plastic deformation that may induce damage, eventually leading to strain localization when material failure is approached. In order to take this phenomenon into account, several theories have been proposed by introducing damage in the constitutive models. The most often used approaches can be classified into two categories: micromechanics-based damage formulations and continuum damage theories. Wellestablished examples of each category are Gurson's damage theory (Gurson, 1977) and the Continuum Damage Mechanics (CDM), popularized by Lemaitre (1985). The first approach consists in describing the material degradation by an internal variable representing the volume fraction of the micro-cavities formed during loading. This approach is widely applied for porous materials (Gurson, 1977; Benallal and Comi, 2000; etc.), as well as for sheet metals (e.g. Brunet and Morestin, 2001). On the other hand, the CDM approach introduces an internal variable that represents the surface density of micro-cracks. It is based on the thermodynamics of the irreversible processes, and is widely applied to metallic materials (Lemaitre and Chaboche, 1985; Kachanov, 1986; Murakami, 1988; Lemaitre, 1992; Voyiadjis and Kattan, 1992a,b; Chaboche, 1999). A comparative study of different ductile models (Lemaitre and Chaboche, 1985; Gurson, 1977; Thomason, 1990; etc) can be found in Pardoen et al. (1998). The CDM approach is adopted in this paper to couple the elastic-plastic model to damage. More specifically, the Teodosiu-Hu hardening model is coupled with a Lemaitre-type damage model. This allows one to simultaneously reproduce strain-path changes and softening effects.

In order to define an intermediate state corresponding to the strain localization during loading, several approaches have been developed in the literature. Some of them are widely applied to determine the FLDs. During the last century, numerous criteria of plastic instabilities have been proposed, e.g. maximum load criteria (Considère, 1885; Swift, 1952; Hora et al., 1996; Brunet and Morestin, 2001), Hill's material stability analysis (Hill, 1952 and 1958), or models assuming an initial defect in the material (Marciniak and Kuczyński, 1967). More 
pragmatic models (e.g. the Marciniak-Kuczyński model) have been given extensive attention because of their industrial applications, in spite of their weaker theoretical foundations (e.g. Kuroda and Tvergaard, 2000a,b, 2004; Aretz, 2008). On the other hand, the theoretically sounder approaches, e.g. Rice's strain localization criterion (Rudnicki and Rice, 1975; Rice, 1976) have mainly been investigated analytically, for simpler constitutive models and for particular (plane stress or plane strain) loading situations (Bigoni and Hueckel, 1991; Doghri and Billardon, 1995; Benallal and Comi, 2000; Benallal and Bigoni, 2004).

In his initial analysis of localized necking, Hill (1952) used a simple constitutive model (rigid-plastic, $J_{2}$ flow theory) with a plane stress condition, showing that localized necking of a thin sheet occurs along the line of minimum straining. As an improvement, Benallal (1998) proposed a three-dimensional analysis of localized necking by studying the properties of the plastic hardening modulus at the point of strain localization with $J_{2}$ elastic-plastic flow theory. Dudzinski and Molinari (1991) proposed a linear perturbation analysis as an alternative to the bifurcation theory to predict plastic instabilities, while Barbier et al. (1998) showed a relation between this approach and the bifurcation one. Hora et al. (1996) proposed an improved version of the Swift criterion and applied it to the prediction of FLDs, while Brunet and Morestin (2001) used this criterion with an advanced elastic-plastic damage constitutive model and finite element analysis. Recently, Kuroda and Tvergaard (2004) studied the development of shear bands by using non-associative plasticity within the framework of a finite element analysis, while Kristensson (2006) used a micromechanical model with void effects (size and distribution in the representative volume element) to study the formability of metal sheets with voids.

However, the Rice localization criterion is seldom applied to study the ductility limit of metal sheets, although its application necessitates only the tangent modulus operator to predict strain localization and the orientation of localization bands. Also, its implementation in a finite element code is relatively easy compared to, for example, the M-K approach.

In this paper, a general and physical approach is proposed to model sheet metal forming by combining: an elastic-plastic constitutive description based on the hardening model of Teodosiu-Hu; a Lemaitre-type isotropic damage model; and Rice's localization criterion. The remainder of the paper is structured in four parts. In the first one (section 2), the complete set of constitutive equations is developed based on the large deformation theory. The second part (section 3) deals with the numerical implementation of the coupled constitutive model. The main aspects of the time integration algorithm are described, and the consistent tangent 
modulus is derived in a compact form in order to implement the model into an implicit finite element (FE) code. In the third part (section 4), the formulation of Rice's localization criterion and its numerical implementation are presented. In the fourth and last part (section 5), the coupled elastic-plastic-damage constitutive model and the localization criterion are applied to predict forming limits for monotonous and two-path straining modes. The ability of the model to reproduce transient features of the hardening and softening due to damage is investigated by means of rheological test simulations. This study allows one to highlight the capability of the proposed approach to predict the FLDs as well as the orientation of the shear bands, during direct and two-step loading paths.

\section{Constitutive equations}

The phenomenological elastic-plastic-damage modeling adopted here is rate independent (without viscous effects) and restricted to cold deformation. The material is initially stressfree (well-annealed state), undamaged and homogeneous. The ingredients of the constitutive model are: a hypo-elastic-damage law defining the stress rate with respect to the elastic strain rate, a yield criterion delimiting the elastic zone, a plastic flow rule, and a set of internal state variable evolution laws defining the work hardening and damage evolution during plastic deformation. Within the framework of large deformation, the constitutive equations require the use of objective rates. The goal of such objective derivatives is to satisfy the material invariance by eliminating all the rotations that do not contribute to the material response. A very common way to deal with this issue is to use the so-called local objective frames associated with various possible decompositions of the deformation gradient, which have been proposed in the literature to extend constitutive material models to the framework of large deformation. Indeed, when writing the constitutive equations in terms of rotation-compensated variables, simple time derivatives are involved in the constitutive equations making them identical in form to a small-strain formulation (see (Haddag et al., 2007) for more details on the large strain kinematics utilized). It should be noted, however, that when the strains become sufficiently large, the choice of the particular co-rotational formulation (Jaumann, Green-Naghdi, plastic spin, etc.) may have an impact on the predicted stress-strain curves and the corresponding limit strains, especially for simple shear. While the Jaumann objective rate is chosen here for simplicity, other methods can be adopted to describe the rotation of the material frame. Another motivation behind this choice is the conformity to Abaqus, which allows our numerical implementation to be validated with regard to existing hardening models that are 
available in Abaqus. Furthermore, it is generally admitted that the particular choice of rotation matrix has little impact within the strain range before localization.

\subsection{Anisotropic elastic-plastic model coupled with damage}

Continuum damage mechanics was first introduced by Kachanov (1958) and slightly modified later by Rabotnov (1969). This concept was then further developed (Lemaitre, 1992; Chaboche, 1999), based on a continuous variable, $d$, related to the density of defects or micro-cracks in the material in order to describe its deterioration. Leckie and Hayhurst (1974), Hult (1974), and Lemaitre and Chaboche (1975) adopted this approach to solve creep and creep-fatigue interaction problems. Later, it was applied to ductile plastic fracture (Lemaitre, 1985) and also to other various applications (Lemaitre, 1984). Note that most of the available presentations on the concept of continuum damage mechanics were concerned with metals as can be found in many treaties and research papers (Altenbach and Skrzypek, 1999; Doghri, 2000; Brünig, 2002; Abu Al-Rub and Voyiadjis, 2003; Brünig, 2003; Brünig et al., 2008). In the context of damage mechanics in metal matrix composites, more recent publications can also be found, which are more specifically devoted to composite materials (see, for instance, Voyiadjis and Kattan, 1999).

Experimental evidence of anisotropy in terms of both plasticity evolution and damage development has been shown (Chow and Wang, 1987a). In this regard, the modeling of anisotropy by using tensor-valued representation of damage has been done e.g. by Lee et al. (1985) and Chow and Wang (1987b), for ductile fracture problems, and more recently by Cicekli et al. (2007), for plain concrete problems. For applications to brittle and creep fracture, a number of contributions using appropriate anisotropic damage models have been published (see e.g. Murakami, 1983; Krajcinovic, 1983).

In the present work, as already announced in the introduction, a general rate-independent, anisotropic, elastic-plastic model is coupled to an isotropic damage model. More precisely, the physically-based hardening model of Teodosiu and Hu (1998) is coupled with the isotropic damage model of Lemaitre (1992). The coupling is carried out through the concept of effective stress, defined as

$$
\tilde{\boldsymbol{\sigma}}=\frac{\boldsymbol{\sigma}}{1-d}
$$


and associated with the principle of strain equivalence (Lemaitre, 1971; Lemaitre and Chaboche, 1985). In this equation, $d$ is the continuum damage variable $(d \in[0,1]$, with $d=0$ for a safe material and $d=1$ for a fully damaged one). This is a scalar variable; thus, damage is assumed to be isotropic, while $\boldsymbol{\sigma}$ is the Cauchy stress tensor in the damaged material and $\tilde{\boldsymbol{\sigma}}$ is the stress tensor in an equivalent undamaged material. This is the classical framework of continuum damage mechanics (Lemaitre and Chaboche, 1985; Lemaitre, 1992; Altenbach and Skrzypek, 1999).

\subsection{Basic equations of the coupled model}

The differentiation of Eq. (1) yields:

$$
\dot{\boldsymbol{\sigma}}=\dot{\tilde{\boldsymbol{\sigma}}}(1-d)-\dot{d} \tilde{\boldsymbol{\sigma}}
$$

As already argued, the forthcoming simple expressions are valid only when applied to rotation-compensated variables, which are adopted throughout this paper. Whenever the stress tensors are expressed in a fixed frame, some objective rates should be used instead.

The rate of the effective stress can be expressed by a hypo-elasticity law:

$$
\dot{\tilde{\boldsymbol{\sigma}}}=\mathbf{C}:\left(\mathbf{D}-\mathbf{D}^{p}\right)
$$

where $\mathbf{C}$ is the fourth-order tensor of the elastic constants, while $\mathbf{D}$ and $\mathbf{D}^{p}$ are second-order tensors denoting the total strain rate and the plastic strain rate, respectively. Replacing in Eq. (2) leads to:

$$
\dot{\boldsymbol{\sigma}}=(1-d) \mathbf{C}:\left(\mathbf{D}-\mathbf{D}^{p}\right)-\dot{d} \tilde{\boldsymbol{\sigma}}
$$

Several approaches have been developed in the literature for coupling this damage description with a plasticity model (Chaboche, 1999). The simplest one is to let damage affect the stress tensor as previously described, while the internal variables describing the hardening remain unaffected. This is the approach used by Lemaitre and co-workers (see Chaboche, 1999; Lemaitre et al., 2000) who coupled damage with several isotropic and/or kinematic hardening models. We adopted the same approach in the current work.

According to Lemaitre (1992), the elastic domain of the damaged material is defined by the yield surface written in the following form:

$$
F=\overline{\tilde{\sigma}}\left(\tilde{\boldsymbol{\sigma}}^{\prime}-\mathbf{X}\right)-Y \leq 0
$$


where $\overline{\tilde{\sigma}}$ is the equivalent effective stress, which is a function of $\tilde{\boldsymbol{\sigma}}^{\prime}=\boldsymbol{\sigma}^{\prime} /(1-d)$ (the deviatoric part of the effective stress), and the back-stress $\mathbf{X}$ (describing the kinematic hardening), whereas $Y$ is the size of the yield surface (related to the isotropic hardening). The associated plastic flow rule reads:

$$
\mathbf{D}^{p}=\dot{\lambda} \frac{\partial F}{\partial \boldsymbol{\sigma}}=\dot{\lambda} \tilde{\mathbf{V}}
$$

where $\tilde{\mathbf{V}}$ is the flow direction normal to the yield surface and $\dot{\lambda}$ is the plastic multiplier that is to be determined from the consistency condition.

By considering the quadratic Hill yield criterion (Hill'48) given by

$$
\overline{\tilde{\sigma}}=\sqrt{\left(\tilde{\boldsymbol{\sigma}}^{\prime}-\mathbf{X}\right): \mathbf{M}:\left(\tilde{\boldsymbol{\sigma}}^{\prime}-\mathbf{X}\right)}
$$

where $\mathbf{M}$ is a fourth-order tensor containing the six anisotropy coefficients of Hill, the plastic flow rule can be expressed as

$$
\mathbf{D}^{p}=\dot{\lambda} \tilde{\mathbf{V}}=\frac{\dot{\lambda}}{(1-d)} \frac{\mathbf{M}:\left(\tilde{\boldsymbol{\sigma}}^{\prime}-\mathbf{X}\right)}{\overline{\tilde{\sigma}}}=\frac{\dot{\lambda}}{(1-d)} \mathbf{V}
$$

The evolution laws of hardening and damage, which will be defined subsequently, make use of the plastic multiplier $\dot{\lambda}$. This latter is related to the equivalent plastic strain rate $\dot{p}$, which is defined as the power conjugate of the equivalent effective stress $\overline{\tilde{\sigma}}$, i.e.

$$
\overline{\tilde{\sigma}} \dot{p}=\left(\tilde{\boldsymbol{\sigma}}^{\prime}-\mathbf{X}\right): \mathbf{D}^{p}
$$

Combining this last equation with Eq. (7), and given the plastic flow rule (8), the relationship between $\dot{p}$ and $\dot{\lambda}$ can be written as

$$
\dot{p}=\frac{\dot{\lambda}}{1-d}
$$

\subsection{Hardening model}

The macroscopic hardening models are based on a set of internal variables, describing the isotropic and kinematic hardening. The Teodosiu-Hu hardening model is described in detail in Teodosiu and $\mathrm{Hu}(1995,1998)$. This model makes use of four internal variables: $\mathbf{X}$ and $R$ are the classical back-stress and isotropic hardening variables, $\mathbf{S}$ is a fourth-order tensor describing the directional strength of the planar persistent dislocation structures, and $\mathbf{P}$ is a second- 
order dimensionless tensor describing the polarity of these structures. In general, kinematic hardening models use either the direction $\mathbf{N}$ of the plastic strain-rate or the direction $\mathbf{v}$ of the deviatoric stress. For a plasticity model coupled to damage, these quantities are defined as:

$$
\tilde{\mathbf{N}}(d)=\frac{\mathbf{D}^{p}}{\left|\mathbf{D}^{p}\right|}=\frac{\mathbf{M}:\left(\tilde{\boldsymbol{\sigma}}^{\prime}-\mathbf{X}\right)}{\left|\mathbf{M}:\left(\tilde{\boldsymbol{\sigma}}^{\prime}-\mathbf{X}\right)\right|}, \quad \tilde{\mathbf{v}}(d)=\frac{\tilde{\boldsymbol{\sigma}}^{\prime}-\mathbf{X}}{\tilde{\tilde{\sigma}}}
$$

For the coupling of the Teodosiu model with damage, the original form of the constitutive equations (Teodosiu and Hu, 1995 and 1998; Haddadi et al., 2006) is kept unchanged, except for the directions $\mathbf{N}$ and $\mathbf{v}$ which are replaced by their "effective" counterparts $\tilde{\mathbf{N}}$ and $\tilde{\mathbf{v}}$.

Part of the isotropic and kinematic hardening is described by classical $R$ and $\mathbf{X}$ variables, like in most combined hardening models:

$$
\begin{gathered}
Y=Y_{0}+R+f|\mathbf{S}| \\
\dot{R}=C_{R}\left(R_{\text {sat }}-R\right) \dot{\lambda}=H_{R} \dot{\lambda} \\
\dot{\mathbf{X}}=C_{X}\left(X_{\text {sat }} \tilde{\boldsymbol{v}}-\mathbf{X}\right) \dot{\lambda}=\mathbf{H}_{\mathbf{X}} \dot{\lambda}
\end{gathered}
$$

The tensor $\mathbf{S}$ is further decomposed along the current plastic strain-rate direction into the scalar, "active" part $S_{D}$ and the fourth-order tensor, "latent" part $\mathbf{S}_{L}$, by the following expressions:

$$
\begin{gathered}
\mathbf{S}=S_{D} \tilde{\mathbf{N}} \otimes \tilde{\mathbf{N}}+\mathbf{S}_{L} \\
S_{D}=\tilde{\mathbf{N}}: \mathbf{S}: \tilde{\mathbf{N}}, \quad \mathbf{S}_{L}=\mathbf{S}-S_{D} \tilde{\mathbf{N}} \otimes \tilde{\mathbf{N}}
\end{gathered}
$$

The evolution laws for $S_{D}, \mathbf{S}_{L}$ and $\mathbf{P}$ are given as:

$$
\begin{gathered}
\dot{S}_{D}=C_{S D}\left[g\left(S_{\text {sat }}-S_{D}\right)-h S_{D}\right] \dot{\lambda}=H_{S_{D}} \dot{\lambda} \\
\dot{\mathbf{S}}_{L}=-C_{S L}\left(\frac{\left|\mathbf{S}_{L}\right|}{S_{s a t}}\right)^{n_{L}} \mathbf{S}_{L} \dot{\lambda}=\mathbf{H}_{\mathbf{S}_{L}} \dot{\lambda} \\
\dot{\mathbf{P}}=C_{p}(\tilde{\mathbf{N}}-\mathbf{P}) \dot{\lambda}=\mathbf{H}_{\mathbf{P}} \dot{\lambda}
\end{gathered}
$$

The functions $g$ and $h$ have been introduced in order to capture transient hardening after a change in strain-path. Their assumed mathematical forms are 


$$
\begin{aligned}
& g= \begin{cases}1-\frac{C_{P}}{C_{S D}+C_{P}}\left|\frac{S_{D}}{S_{s a t}}-\mathbf{P}: \tilde{\mathbf{N}}\right| & \text { if } \mathbf{P}: \tilde{\mathbf{N}} \geq 0 \\
(1+\mathbf{P}: \tilde{\mathbf{N}})^{n_{p}}\left(1-\frac{C_{P}}{C_{S D}+C_{P}} \frac{S_{D}}{S_{s a t}}\right) & \text { otherwise }\end{cases} \\
& h=\frac{1}{2}\left(1-\frac{\mathbf{X}: \tilde{\mathbf{N}}}{X_{s a t} \tilde{\mathbf{n}}: \tilde{\mathbf{N}}}\right)
\end{aligned}
$$

and $X_{\text {sat }}$ is a function of $\mathbf{S}$, given by

$$
X_{s a t}=X_{0}+(1-f) \sqrt{r|\mathbf{S}|^{2}+(1-r) S_{D}^{2}}
$$

Consequently, this hardening model involves 13 parameters: $Y_{0}, R_{s a t}, C_{R}, X_{0}, C_{X}, C_{P}$, $S_{s a t}, C_{S D}, C_{S L}, n_{L}, n_{p}, r$ and $f$. The identification of these hardening parameters is a difficult task. The experiments required for the identification include monotonic tensile and shear tests, but also two or three reverse (preferably shear) tests and at least one orthogonal test (e.g. tensile test followed by shear test). The sensitivity of the predicted stress-strain curves to some of the hardening model parameters is restricted to small zones of one or several curves (e.g. the transition zone of one or the other of the sequential tests). A detailed parameter identification method for this particular hardening model has been described by Haddadi et al. (2006) and several sets of material parameters are provided therein for several sheet metals. The material parameters used in the current paper are selected from Haddadi et al. (2006).

It is noteworthy that although the adopted coupling with a relatively basic isotropic damage model modifies the equations, their mathematical structure remains identical to their uncoupled form (see original work of Teodosiu and $\mathrm{Hu}, 1998$ ). Indeed, the assumption of a scalar damage variable is convenient for its simplicity and allows the derivation of the coupled evolution equations to be more easily handled. Besides this simplicity and the abovementioned difficulties related to material parameter identification, the seemingly formidentical mathematical structure for the constitutive equations is another motivation that justifies the choice of this simple approach for damage. This property proved very useful for the numerical implementation of the coupled model in a finite element code. 


\subsection{Damage evolution law}

Several damage evolution laws are proposed in the literature within the framework of the so-called continuum damage mechanics (Lemaitre, 1992; Lemaitre et al., 2000; Hammi, 2000, etc.), especially for sheet metals. Here, the evolution law of the damage variable $d$ is assumed to be of the following form:

$$
\dot{d}=H_{d} \dot{\lambda}=\left\{\begin{array}{lc}
\frac{1}{(1-d)^{\beta}}\left(\frac{Y^{e}-Y_{i}^{e}}{s}\right)^{s} \dot{\lambda} \quad \text { if } & Y^{e} \geq Y_{i}^{e} \\
0 & \text { else }
\end{array}\right.
$$

which was recently used by Khelifa (2004) to predict damage in deep drawing process simulations. The scalar quantities $s, S, \beta$ and $Y_{i}^{e}$ are material parameters, while the so-called strain energy density release rate $Y^{e}$ is given, for linear isotropic elasticity, by the following phenomenological expression (see e.g. Lemaitre and Chaboche, 1985; Lemaitre, 1992):

$$
Y^{e}=\frac{\tilde{J}_{2}^{2}}{2 E}\left[\frac{2}{3}(1+v)+3(1-2 v)\left(\frac{\tilde{\sigma}^{s}}{\tilde{J}_{2}}\right)^{2}\right]
$$

where $\tilde{\sigma}^{s}=\frac{1}{3} \operatorname{tr}(\tilde{\boldsymbol{\sigma}})$ is the hydrostatic effective stress, $\tilde{J}_{2}=\sqrt{\frac{3}{2} \tilde{\boldsymbol{\sigma}}^{\prime}: \tilde{\boldsymbol{\sigma}}^{\prime}}$ is the second invariant of the effective stress deviator, and $E$ and $v$ are, respectively, the Young modulus and Poisson ratio of the undamaged material. Note that Germain et al. (1983) were the first to suggest the extension of Eq. (22) from a linear relation to a more general power law $(s>1)$.

\subsection{Analytical tangent modulus}

Usually, rate-independent elastic-plastic laws can be written in the following compact form:

$$
\dot{\boldsymbol{\sigma}}=\mathbf{L}^{\text {ana }}: \mathbf{D}
$$

where $\mathbf{L}^{\text {ana }}$ is the so-called analytical tangent modulus. Again, note that this equation is written in the local (material) frame. The expression of this modulus is required when formulating the Rice localization criterion (see section 4) for the chosen material.

Let us first determine the plastic multiplier $\dot{\lambda}$. The consistency condition $\dot{F}=0$ leads to

$$
\dot{\tilde{\sigma}}-\dot{Y}=0
$$


The first term is obtained by the chain rule as

$$
\dot{\overline{\tilde{\sigma}}}=\frac{\partial \overline{\tilde{\sigma}}}{\partial \tilde{\boldsymbol{\sigma}}^{\prime}}: \dot{\tilde{\boldsymbol{\sigma}}}^{\prime}+\frac{\partial \overline{\tilde{\sigma}}}{\partial \mathbf{X}}: \dot{\mathbf{X}}=\frac{\partial \overline{\tilde{\sigma}}}{\partial \tilde{\boldsymbol{\sigma}}^{\prime}}:\left(\dot{\tilde{\boldsymbol{\sigma}}}^{\prime}-\dot{\mathbf{X}}\right)
$$

and its subsequent terms are obtained as follows:

$$
\begin{gathered}
\dot{\tilde{\boldsymbol{\sigma}}}^{\prime}=\mathbf{C}:\left(\mathbf{D}^{\prime}-\mathbf{D}^{p}\right)=\mathbf{C}:\left(\mathbf{D}^{\prime}-\tilde{\mathbf{V}} \dot{\lambda}\right) \\
\frac{\partial \overline{\tilde{\sigma}}}{\partial \tilde{\boldsymbol{\sigma}}^{\prime}}=\frac{\mathbf{M}:\left(\tilde{\boldsymbol{\sigma}}^{\prime}-\mathbf{X}\right)}{\tilde{\tilde{\sigma}}}=\mathbf{V} \\
\dot{\mathbf{X}}=\mathbf{H}_{\mathbf{x}} \dot{\lambda} \\
\dot{Y}=H_{Y} \dot{\lambda}
\end{gathered}
$$

Back-substitution of these results leads to the following expression for the plastic multiplier:

$$
\dot{\lambda}=\frac{\mathbf{V}: \mathbf{C}: \mathbf{D}^{\prime}}{H_{\lambda}}=\frac{\mathbf{V}: \mathbf{C}: \mathbf{D}}{H_{\lambda}}
$$

where $H_{\lambda}$ is the scalar hardening modulus, affected by damage:

$$
H_{\lambda}=\mathbf{V}: \mathbf{C}: \tilde{\mathbf{V}}+\mathbf{V}: \mathbf{H}_{\mathbf{x}}+H_{Y}
$$

Finally, after substituting in Eq. (4) and rearranging all the terms, the following linear rate equation is found:

$$
\dot{\boldsymbol{\sigma}}=\left[\tilde{\mathbf{C}}-\alpha\left(\frac{(\mathbf{C}: \mathbf{V}) \otimes(\mathbf{V}: \mathbf{C})}{H_{\lambda}}+\frac{H_{d} \tilde{\boldsymbol{\sigma}} \otimes(\mathbf{V}: \mathbf{C})}{H_{\lambda}}\right)\right]: \mathbf{D}
$$

where $\alpha=1$ for plastic loading and 0 otherwise, and $\tilde{\mathbf{C}}=(1-d) \mathbf{C}$.

When the tensor $\mathbf{C}$ is isotropic, these expressions are further simplified, giving

$$
\dot{\lambda}=\frac{2 G \mathbf{V}: \mathbf{D}}{H_{\lambda}}
$$

and

$$
\mathbf{L}^{a n a}=\tilde{\mathbf{C}}-\alpha\left(\frac{4 G^{2}(1-d)^{2} \tilde{\mathbf{V}} \otimes \tilde{\mathbf{V}}}{H_{\lambda}}+\frac{2 G H_{d} \boldsymbol{\sigma} \otimes \tilde{\mathbf{V}}}{H_{\lambda}}\right)
$$


where $G$ is the shear modulus and $H_{\lambda}$ is given by

$$
H_{\lambda}=(1-d)\left(2 G \tilde{\mathbf{V}}: \tilde{\mathbf{V}}+\tilde{\mathbf{V}}: \mathbf{H}_{\mathbf{x}}\right)+H_{Y}
$$

One can notice that, if there is no damage in the model, then $\tilde{\mathbf{C}}=\mathbf{C}, \tilde{\mathbf{V}}=\mathbf{V}, \tilde{\boldsymbol{\sigma}}=\boldsymbol{\sigma}$ and $H_{d}$ vanishes; in this case, it is easy to see that the classical expression of the elastic-plastic tangent modulus is recovered (see e.g. Haddag et al., 2007).

\section{Numerical implementation}

In a finite element code, the constitutive model takes the form of a stress (and state) update scheme between a time $t$ (at increment $n$ ) and the subsequent time $t+\Delta t$ (increment $n+1)$. The numerical implementation of this coupled model is done here using an implicit time integration scheme, following the Hughes and Winget (1980) approach, i.e. integration of the constitutive equations in the co-rotational frame. For the selected elastic-plastic model without damage, an accurate, implicit state update algorithm has already been developed (Haddag et al., 2007). The numerical implementation of the damage-coupled model follows the same approach (see e.g. Benallal et al., 1988) and, under some assumptions, it will take a similar form.

First, the discrete forms of the constitutive equations are reviewed. Then, the main steps of the derivation of the consistent (algorithmic) tangent modulus are given.

\subsection{Discrete form of the constitutive equations}

Elasticity and normality laws. The discrete form of the elasticity law is

$$
\Delta \tilde{\boldsymbol{\sigma}}=\mathbf{C}: \Delta \boldsymbol{\varepsilon}^{e}=\mathbf{C}:\left(\Delta \boldsymbol{\varepsilon}-\Delta \boldsymbol{\varepsilon}^{p}\right)
$$

This leads to the stress update equation

$$
\boldsymbol{\sigma}_{n+1}=\left(1-d_{n+1}\right)\left[\tilde{\mathbf{\sigma}}_{n}+\mathbf{C}:\left(\Delta \boldsymbol{\varepsilon}-\Delta \boldsymbol{\varepsilon}^{p}\right)\right]
$$

where $\tilde{\boldsymbol{\sigma}}_{n}=\boldsymbol{\sigma}_{n} /\left(1-d_{n}\right)$. It appears that the final stress depends on the plastic strain increment and on the damage. The plastic strain increment is obtained from the discrete normality rule:

$$
\Delta \boldsymbol{\varepsilon}^{p}=\left.\Delta \lambda \frac{\partial F}{\partial \boldsymbol{\sigma}}\right|_{n+1}=\tilde{\mathbf{V}}_{n+1} \Delta \lambda=\frac{1}{1-d_{n+1}} \mathbf{V}_{n+1} \Delta \lambda
$$


where the implicit character of the scheme clearly appears.

Hardening variables. The hardening variables are governed by rate equations of the form $\dot{\mathbf{y}}=\mathbf{H}_{\mathrm{y}} \dot{\lambda}$. According to Haddag et al. (2007), they are updated with an implicit, semianalytical scheme. The following form of the update equations is obtained

$$
\mathbf{y}_{n+1}=\mathbf{y}_{n}+\Delta \mathbf{y}\left(\boldsymbol{\sigma}_{n+1}, \mathbf{y}_{n+1}, \Delta \lambda\right)
$$

Damage. The semi-analytical time integration approach already used in Haddag et al. (2006, 2007) is used for the damage variable, which leads to

$$
\begin{array}{ll}
d_{n+1}=1-\left[\left(1-d_{n}\right)^{1+\beta}-(1+\beta)\left(\frac{Y_{n+1}^{e}-Y_{i}^{e}}{S}\right)^{s} \Delta \lambda\right]^{\frac{1}{1+\beta}} & \text { if } Y_{n+1}^{e} \geq Y_{i}^{e} \\
d_{n+1}=d_{n} & \text { otherwise }
\end{array}
$$

\subsection{Numerical resolution}

The resolution of the previous set of nonlinear equations is performed using the NewtonRaphson procedure. In Haddag et al. (2007), the elastic-plastic model has been reduced to a set of two equations, depending only on the main variables $\mathbf{T}=\boldsymbol{\sigma}^{\prime}-\mathbf{X}$ and $\Delta \lambda$. The size reduction of the linear systems is a common preoccupation when constitutive models are implemented (see e.g. Alves, 2003; Khelifa, 2004; Haddag et al., 2007, etc.). This approach ensures the robustness of the Newton-Raphson resolution, while keeping computing times within reasonable values. In the case of the damage-coupled model, adopting the same reduction procedure leads to the following two-equation system:

$$
\left[\begin{array}{l}
\tilde{\mathbf{T}}_{n+1}-\tilde{\mathbf{T}}_{n}-2 G \Delta \boldsymbol{\varepsilon}^{\prime}+2 G \Delta \lambda \tilde{\mathbf{V}}\left(\tilde{\mathbf{T}}_{n+1}, d_{n+1}\right)+\Delta \mathbf{X}\left(\tilde{\mathbf{T}}_{n+1}, \Delta \lambda\right) \\
\tilde{\tilde{\sigma}}\left(\tilde{\mathbf{T}}_{n+1}\right)-Y\left(\tilde{\mathbf{T}}_{n+1}, \Delta \lambda\right)
\end{array}\right]=\left[\begin{array}{l}
\mathbf{0} \\
0
\end{array}\right]
$$

where $\tilde{\mathbf{T}}=\tilde{\boldsymbol{\sigma}}^{\prime}-\mathbf{X}$ and $\Delta \boldsymbol{\varepsilon}^{\prime}$ is the deviatoric part of the strain increment. Nevertheless, Eq. (41) should be added to this nonlinear system, which can be subsequently solved for $\tilde{\mathbf{T}}_{n+1}, \Delta \lambda$ and $d_{n+1}$. For the current implementation, a simpler approach has been chosen upon noticing that the damage variable $d_{n+1}$ enters the system (42) only through the $\tilde{\mathbf{V}}$ variable. Therefore, the damage equation is uncoupled for this system at each increment by considering the value $d_{n}$ instead of $d_{n+1}$ wherever it explicitly appears in system (42), namely in only one term ( $\left.\tilde{\mathbf{V}}\right)$. 
By doing this, the numerical resolution becomes very similar to the one used for the undamaged model. Another motivation behind this assumption is that the damage equation exhibits a yield value; thus, during the first part of the loading history, the previous approximation will have no impact on the solution. During the last part, when damage is activated, the strain increments should be restricted to safe values in order to ensure the overall accuracy.

\subsection{Consistent tangent modulus}

For the finite element equilibrium resolution, the constitutive algorithm must also provide the variation of the stress increment due to a variation in the strain increment:

$$
D(\Delta \boldsymbol{\sigma})=\mathbf{L}^{a l g}: D(\Delta \boldsymbol{\varepsilon})
$$

The fourth-order tensor $\mathbf{L}^{\text {alg }}$ is the so-called consistent tangent modulus (algorithmic modulus), which is calculated hereafter.

The differentiation of the elasticity law gives

$$
D(\Delta \boldsymbol{\sigma})=(1-d) \mathbf{C}: D(\Delta \boldsymbol{\varepsilon})-(1-d) 2 G D\left(\Delta \boldsymbol{\varepsilon}^{p}\right)-\tilde{\boldsymbol{\sigma}} D(d)
$$

The differential of the plastic strain increment, $D\left(\Delta \boldsymbol{\varepsilon}^{p}\right)$, can be determined by differentiation of the normality rule:

$$
D\left(\Delta \boldsymbol{\varepsilon}^{p}\right)=\Delta \lambda D(\tilde{\mathbf{V}})+\tilde{\mathbf{V}} D(\Delta \lambda)
$$

The differentiation of the yield criterion and $\tilde{\mathbf{V}}$, respectively, gives

$$
D(\Delta \lambda)=\frac{1}{H_{Y}^{d}}\left(\mathbf{V}-\frac{\partial Y}{\partial \tilde{\mathbf{T}}}\right): D(\tilde{\mathbf{T}}), \quad D(\tilde{\mathbf{V}})=\tilde{\mathbf{Q}}: D(\tilde{\mathbf{T}})
$$

where

$$
\tilde{\mathbf{Q}}=\frac{\partial \tilde{\mathbf{V}}}{\partial \tilde{\mathbf{T}}}=\frac{1}{(1-d) \overline{\tilde{\sigma}}}(\mathbf{M}-\mathbf{V} \otimes \mathbf{V}), \quad H_{Y}^{d}=\frac{\partial Y}{\partial \Delta \lambda}
$$

By replacing $D(\Delta \lambda)$ and $D(\tilde{\mathbf{V}})$ in Eq. (45) and then in Eq. (44), one can obtain

$$
D(\Delta \boldsymbol{\sigma})=(1-d) \mathbf{C}: D(\Delta \boldsymbol{\varepsilon})-(1-d) 2 G\left[\frac{1}{H_{Y}^{d}} \tilde{\mathbf{V}} \otimes\left(\mathbf{V}-\frac{\partial Y}{\partial \tilde{\mathbf{T}}}\right)+\Delta \lambda \tilde{\mathbf{Q}}\right]: D(\tilde{\mathbf{T}})-\tilde{\boldsymbol{\sigma}} D(d)
$$


One still has to express $D(\tilde{\mathbf{T}})=D\left(\tilde{\boldsymbol{\sigma}}^{\prime}\right)-D(\mathbf{X})$. By differentiating $\tilde{\boldsymbol{\sigma}}^{\prime}$ and $\mathbf{X}$, and replacing in the relation of $D(\tilde{\mathbf{T}})$, one can obtain

$$
D(\tilde{\mathbf{T}})=2 G \boldsymbol{\Lambda}^{-1}: D(\Delta \boldsymbol{\varepsilon})
$$

with

$$
\boldsymbol{\Lambda}=\mathbf{I}_{4}^{\prime}+2 G\left[\frac{1}{H_{Y}^{d}} \tilde{\mathbf{V}} \otimes\left(\mathbf{V}-\frac{\partial Y}{\partial \tilde{\mathbf{T}}}\right)+\Delta \lambda \tilde{\mathbf{Q}}\right]+\frac{\partial \mathbf{X}}{\partial \tilde{\mathbf{T}}}+\frac{1}{H_{Y}^{d}} \frac{\partial \mathbf{X}}{\partial \Delta \lambda} \otimes\left(\mathbf{V}-\frac{\partial Y}{\partial \tilde{\mathbf{T}}}\right)
$$

where $\mathbf{I}_{4}^{\prime}$ is the fourth-order symmetric and deviatoric identity tensor. By differentiating the discrete form of the damage evolution law (Eq. (41)), $D(d)$ reads

$$
D(d)=\frac{\partial d}{\partial \Delta \lambda} D(\Delta \lambda)+\frac{\partial d}{\partial Y^{e}} D\left(Y^{e}\right)
$$

The differentiation of Eq. (23) gives

$$
D\left(Y^{e}\right)=\frac{1}{2 G} \tilde{\boldsymbol{\sigma}}^{\prime}:\left\{\mathbf{C}-4 G^{2}\left[\frac{1}{H_{Y}^{d}} \tilde{\mathbf{V}} \otimes\left(\mathbf{V}-\frac{\partial Y}{\partial \tilde{\mathbf{T}}}\right)+\Delta \lambda \tilde{\mathbf{Q}}\right]: \boldsymbol{\Lambda}^{-1}\right\}: D(\Delta \boldsymbol{\varepsilon})+3 \tilde{\boldsymbol{\sigma}}^{s}: D(\Delta \boldsymbol{\varepsilon})
$$

By replacing all these terms, $D(\Delta \boldsymbol{\sigma})$ can be linearly related to $D(\Delta \boldsymbol{\varepsilon})$ (see Eq. (43)) using the consistent modulus $\mathbf{L}^{\text {alg }}$, which takes the following form:

$$
\begin{aligned}
D(\Delta \boldsymbol{\sigma}) & =(1-d)\left\{\mathbf{C}-4 G^{2}\left[\frac{1}{H_{Y}^{d}} \tilde{\mathbf{V}} \otimes\left(\mathbf{V}-\frac{\partial Y}{\partial \tilde{\mathbf{T}}}\right)+\Delta \lambda \tilde{\mathbf{Q}}\right]: \boldsymbol{\Lambda}^{-1}\right\}: D(\Delta \boldsymbol{\varepsilon}) \\
& -\frac{\partial d}{\partial Y^{e}} \frac{1}{2 G} \tilde{\boldsymbol{\sigma}} \otimes \tilde{\boldsymbol{\sigma}}^{\prime}:\left\{\mathbf{C}-4 G^{2}\left[\frac{1}{H_{Y}^{d}} \tilde{\mathbf{V}} \otimes\left(\mathbf{V}-\frac{\partial Y}{\partial \tilde{\mathbf{T}}}\right)+\Delta \lambda \tilde{\mathbf{Q}}\right]: \boldsymbol{\Lambda}^{-1}\right\}: D(\Delta \boldsymbol{\varepsilon}) \\
& -\frac{\partial d}{\partial Y^{e}} 3 \tilde{\boldsymbol{\sigma}} \otimes \tilde{\boldsymbol{\sigma}}^{s}: D(\Delta \boldsymbol{\varepsilon})-\frac{2 G}{H_{Y}^{d}} \frac{\partial d}{\partial \Delta \lambda} \tilde{\boldsymbol{\sigma}} \otimes\left(\mathbf{V}-\frac{\partial Y}{\partial \tilde{\mathbf{T}}}\right): \boldsymbol{\Lambda}^{-1}: D(\Delta \boldsymbol{\varepsilon})
\end{aligned}
$$

This last expression defines the algorithmic tangent modulus $\mathbf{L}^{\text {alg }}$ to be used for the numerical implementation into an implicit finite element code.

\section{Localization criterion}

As mentioned in the introduction, several theories have been proposed in the literature to predict strain localization in sheet metals. One of the most popular theories is the Marciniak- 
Kuczyński analysis, which has been intensively used in sheet metal forming, both due to its simple implementation and also for its applicability to a wide range of materials: ratedependent and rate-independent behavior models. The localization criterion first proposed by Rice (Rudnicki and Rice, 1975; Rice, 1976) is used in this study. This criterion has already been applied by other authors to analyze strain localization for different material behavior models. For example, Benallal and Comi (2000) applied this criterion to porous media, Lemaitre et al. (2000) used it to define a critical value of damage in an elastic-plastic-damaged material, while Doghri and Billardon (1995) adopted this criterion to predict the orientation of the localization bands for an elastic-plastic-damaged material within a finite element analysis. In a recent study, this bifurcation criterion has also been used with a Gurson-type model for ductile fracture analysis (Sánchez et al., 2008). Ito and co-workers (Ito et al., 2000) have combined an original constitutive law that takes into account the fact that "the direction of the stress rate affects the direction of the plastic strain rate" (Goya and Ito, 1990) and Hill's quadratic yield surface with Rice's criterion to compute FLDs without pre-strain. Recently, Rice's

criterion has been combined with a self-consistent micromechanical model to predict ductility limits (Franz et al., 2008, 2009), while Signorelli et al. (2008) and Wu et al. (2005, 2007) used the M-K approach combined with crystal plasticity models.

In addition to its sound mathematical background, this criterion does not require any fitting parameter and it is independent of the resolution of the constitutive equations. Therefore, it is relatively easy to implement as a post-processing computation. Moreover, it predicts the limit strains reached at localization, as well as the orientation of the localization band. Rice (1976) indicates that the criterion does not detect any strain localization in the case of associative plasticity, unless a softening behavior is considered in the constitutive model.

The basic ideas underlying this criterion are briefly recalled hereafter, before the particular form of the analytical tangent modulus required for its application is derived.

\subsection{Rice's localization criterion}

This criterion applies to a continuous medium undergoing a homogeneous strain state. The strain localization is searched for as a bifurcation phenomenon, meaning that a nonhomogeneous straining mode becomes possible (i.e., the uniqueness of the solution of the rate equations is lost). This non-homogeneity is considered as a planar localization band, defined by its normal $\mathbf{n}$ (see Fig. 1). The velocity gradient inside and outside the band are respectively denoted as $\mathbf{G}^{-}$and $\mathbf{G}^{+}$, while the corresponding nominal stresses are denoted as $\boldsymbol{\Sigma}^{-}$and $\boldsymbol{\Sigma}^{+}$. 


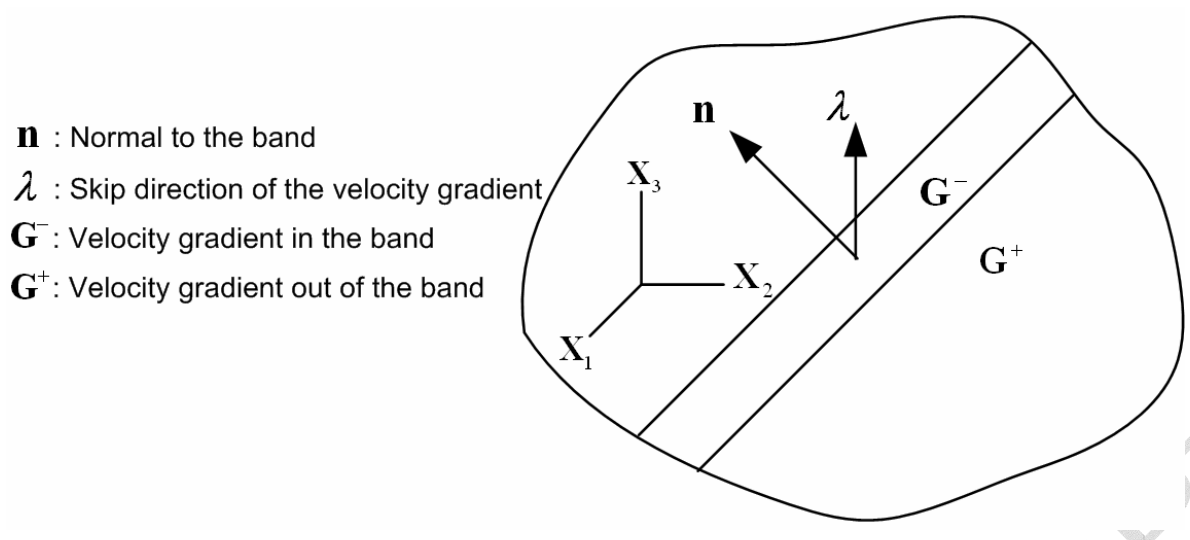

Fig. 1. Schematic representation of the localization band.

The nominal stress rate is related to the velocity gradient by the following constitutive law:

$$
\dot{\Sigma}=L: \mathbf{G}
$$

where $\boldsymbol{L}$ is an analytical tangent modulus that has to be expressed in terms of $\mathbf{L}^{\text {ana }}$ and other fourth-order moduli induced by the large strain formalism. The continuity of the stress vector through the band of normal $\mathbf{n}$ is written as

$$
\mathbf{n} \cdot[\dot{\boldsymbol{\Sigma}}]=\mathbf{0}
$$

where $[\mathbf{A}]=\mathbf{A}^{+}-\mathbf{A}^{-}$designates the jump in a quantity $\mathbf{A}$ across the chosen plane. Maxwell's compatibility condition for the velocity field states that a vector $\lambda$ exists such that the jump in G reads

$$
[\mathbf{G}]=\lambda \otimes \mathbf{n}
$$

Note that $\lambda=\mathbf{0}$ enforces a continuous velocity gradient field. Combining Eqs. (54)-(56), one obtains

$$
(\mathbf{n} \cdot \boldsymbol{L} \cdot \mathbf{n}) \cdot \boldsymbol{\lambda}=\mathbf{0}
$$

This is a typical eigenvalue problem and the existence of a nontrivial solution for $\lambda$ (i.e., bifurcation condition $\boldsymbol{\lambda} \neq \mathbf{0}$ ) requires that the following determinant vanishes:

$$
\operatorname{det}(\mathbf{n} \cdot \boldsymbol{L} \cdot \mathbf{n})=0
$$


This last equation gives a necessary condition for a localization band to appear (i.e., when the acoustic tensor becomes singular, thus corresponding also to the loss of ellipticity of the boundary value problem); it describes the strain localization criterion introduced by Rice (1976).

\subsection{Tangent modulus for the ellipticity loss prediction}

The application of the former localization criterion requires the calculation of the modulus $\boldsymbol{L}$. In a fixed frame, the hypo-elasticity law reads

$$
\ddot{\boldsymbol{\sigma}}=\mathbf{C}:\left(\mathbf{D}-\mathbf{D}^{p}\right)
$$

where $\tilde{\tilde{\boldsymbol{\sigma}}}$ designates the Jaumann derivative of the effective Cauchy stress

$$
\stackrel{\tilde{\boldsymbol{\sigma}}}{=} \dot{\tilde{\boldsymbol{\sigma}}}-\mathbf{W} \cdot \tilde{\boldsymbol{\sigma}}+\tilde{\boldsymbol{\sigma}} \cdot \mathbf{W}
$$

The stress rate can be thus expressed as

$$
\dot{\boldsymbol{\sigma}}=(1-d) \tilde{\boldsymbol{\sigma}}-\dot{d} \tilde{\boldsymbol{\sigma}}+\mathbf{W} \cdot \boldsymbol{\sigma}-\boldsymbol{\sigma} \cdot \mathbf{W}
$$

The Cauchy stress and the nominal stress are related to each other by the classical relation

$$
J \boldsymbol{\sigma}=\mathbf{F} \cdot \boldsymbol{\Sigma}
$$

where $\mathbf{F}$ is the deformation gradient and $J=\operatorname{det}(\mathbf{F})$ is its Jacobian. Thus,

$$
\dot{\boldsymbol{\Sigma}}=J \mathbf{F}^{-1} \cdot(\boldsymbol{\sigma} \operatorname{tr}(\mathbf{D})+\dot{\boldsymbol{\sigma}}-\mathbf{G} \cdot \boldsymbol{\sigma})
$$

In an updated Lagrangian formulation, $\mathbf{F}=\mathbf{I}$ and $J=1$; Eq. (63) simplifies as:

$$
\dot{\boldsymbol{\Sigma}}=\dot{\boldsymbol{\sigma}}+\boldsymbol{\sigma} \operatorname{tr}(\mathbf{D})-\mathbf{G} \cdot \boldsymbol{\sigma}
$$

and, replacing the Cauchy stress rate from Eq. (61) yields:

$$
\dot{\boldsymbol{\Sigma}}=(1-d) \mathbf{C}:\left(\mathbf{D}-\mathbf{D}^{p}\right)-\dot{d} \tilde{\boldsymbol{\sigma}}-\boldsymbol{\sigma} \cdot \mathbf{W}+\boldsymbol{\sigma} \operatorname{tr}(\mathbf{D})-\mathbf{D} \cdot \boldsymbol{\sigma}
$$

All terms on the right-hand side of this above expression can be linearly expressed in terms of the velocity gradient $\mathbf{G}$, in the following way:

$$
\begin{gathered}
(1-d) \mathbf{C}:\left(\mathbf{D}-\mathbf{D}^{p}\right)-\dot{d} \tilde{\boldsymbol{\sigma}}=\mathbf{L}^{a n a}: \mathbf{G} \\
\boldsymbol{\sigma} \operatorname{tr}(\mathbf{D})=\mathbf{L}_{1}: \mathbf{G}
\end{gathered}
$$




$$
\begin{aligned}
& \mathbf{D} \cdot \boldsymbol{\sigma}=\mathbf{L}_{2}: \mathbf{G} \\
& \boldsymbol{\sigma} \cdot \mathbf{W}=\mathbf{L}_{3}: \mathbf{G}
\end{aligned}
$$

where $\mathbf{L}^{\text {ana }}$ is the analytical tangent modulus from Eqs. (24) and (33), while $\mathbf{L}_{1}, \mathbf{L}_{2}$ and $\mathbf{L}_{3}$ are fourth-order tensors that can be expressed, after some mathematical manipulations, as

$$
\begin{gathered}
L_{1 i j k l}=\sigma_{i j} \delta_{k l} \\
L_{2 i j k l}=\frac{1}{2}\left[\delta_{i k} \sigma_{l j}+\delta_{i l} \sigma_{k j}\right] \\
L_{3 i j k l}=\frac{1}{2}\left[\sigma_{i k} \delta_{l j}-\sigma_{i l} \delta_{j k}\right]
\end{gathered}
$$

and finally

$$
\mathbf{L}=\mathbf{L}^{\text {ana }}+\mathbf{L}_{1}-\mathbf{L}_{2}-\mathbf{L}_{3}
$$

It is noteworthy that the resulting modulus $L$ possesses no symmetry, due to the particular forms of the three $\mathbf{L}_{i}$ terms.

\subsection{Numerical implementation of the localization criterion}

The numerical detection of localization with Rice's criterion can be considered as a minimization problem:

$$
\left\{\begin{array}{l}
\operatorname{minimize} f(\mathbf{n}) \text { with }|\mathbf{n}|=1 \\
f(\mathbf{n})=\operatorname{det}\{\mathbf{n} \cdot \boldsymbol{L} \cdot \mathbf{n}\}
\end{array}\right.
$$

In the case where $\min [f(\mathbf{n})]>0$, there is no localization in the material point considered. Otherwise, $\exists \mathbf{n}_{\text {loc }} / f\left(\mathbf{n}_{\text {loc }}\right)=0$, corresponding to the moment of the apparition of localization, where $\mathbf{n}_{l o c}$ represents all possible normal directions to the bands so formed. The applied algorithm is composed of the following main steps:

1. Compute the tangent modulus $L$ at the end of the loading increment.

2. Compute $f(\mathbf{n})$ for different directions.

3. Search the direction $\mathbf{n}_{\min }$, thereby giving the minimum of $f(\mathbf{n})$.

4. Verify whether $f\left(\mathbf{n}_{\text {min }}\right) \leq 0$. 
$\bigcirc \quad$ If .true. then $\mathbf{n}_{l o c}=\mathbf{n}_{\text {min }}$ is the orientation of the first band and strain localization is reached.

- Otherwise, continue to 1 for the next loading increment.

\section{Applications}

In this section, the capability of the proposed approach to predict the FLDs, as well as the orientation of the shear bands, in monotonic and two-step sequential loading paths, is investigated. The study highlights the capability of the coupled model to predict different characteristics of the stress-strain relationship, especially its potential to simultaneously reproduce transient features of the hardening due to strain-path changes and the softening due to damage.

\subsection{Validation of the constitutive model}

Several direct and sequential loadings are considered for the investigation of the constitutive model and the validation of the computer implementation. The simulated tests correspond to typical in-plane sheet metal rheological tests, which are regularly used for the experimental and numerical assessment of FLDs using various models.

The materials used for the investigations are a dual phase high strength steel and a mild steel. The corresponding plastic anisotropy and hardening parameters (Hill'48 yield surface and Teodosiu-Hu hardening parameters) are listed in Table 1 and have been taken from Haddadi et al. (2006) and (3DS Report, 2001). The initial anisotropy is rather negligible for the dual phase steel ( $F, G$, and $H$ close to 0.5$)$, while it is important for the mild steel. Plastic anisotropy is known to dramatically influence the forming limits and thus the use of the anisotropic Hill criterion is compulsory for the mild steel. Concerning hardening parameters, it is noteworthy that the latent part of the directional strength tensor, $\mathbf{S}_{L}$, is deactivated for the dual phase steel, since $C_{S L}$ and $r$ are set to zero.

In (Haddadi et al., 2006), the material parameters are also available for a simpler hardening model combining the Armstrong-Frederick kinematic hardening model, Eq. (14) without coupling to damage (i.e., $d=0, \tilde{\boldsymbol{\sigma}}=\boldsymbol{\sigma}$, and $\tilde{\boldsymbol{v}}=v$ ), with the Swift isotropic hardening model:

$$
R=C_{R}\left(\varepsilon_{0}+\bar{\varepsilon}^{p}\right)^{n}
$$


where $C_{R}, \varepsilon_{0}$ and $n$ are material parameters and $\bar{\varepsilon}^{p}$ is the cumulated plastic strain. This classical model will be used hereafter as a reference when useful; the corresponding parameters are also listed in Table 1.

One should note, however, that coupling with damage was not taken into account during the parameter identification of the elastic-plastic model. Parameter identification of damage models is a troublesome task and, to the knowledge of the authors, no attempt has been made to identify the parameters for the Teodosiu model coupled with damage. Moreover, the hardening parameters also depend on the parameter identification of the yield function, which also may influence considerably the FLD (Aretz, 2007). For the current work, a numerical study has been performed to address the impact of the damage parameters on the stress-strain curves and the FLDs. Consequently, a set of parameters has been chosen (see Table 2) that exhibits acceptable results, e.g., in terms of limit strains for simple tests. While one cannot consider the resulting set of parameters to correspond to the real materials under study, the use of the same set of damage parameters for both the Teodosiu model and the reference ArmstrongFrederick-Swift (AFS) model is believed to be consistent for the numerical comparison performed in the remainder of the paper. 
Table 1. Parameters of plastic anisotropy and hardening for the two materials analyzed in the paper, from Haddadi et al. (2006).

\begin{tabular}{|c|c|c|c|c|c|}
\hline \multicolumn{3}{|c|}{ Hill'48 yield function parameters } & \multicolumn{3}{|c|}{ Teodosiu model parameters } \\
\hline & dual phase & mild steel & & dual phase & mild steel \\
\hline $\mathrm{F}$ & 0.490 & 0.251 & $Y_{0}(\mathrm{MPa})$ & 308.3 & 122.2 \\
\hline G & 0.504 & 0.297 & $C_{R}$ & 49.7 & 27.3 \\
\hline $\mathrm{H}$ & 0.496 & 0.703 & $R_{s a t}(\mathrm{MPa})$ & 125.2 & 80 \\
\hline $\mathrm{L}$ & 1.5 & 1.5 & $C_{X}$ & 53.5 & 614.6 \\
\hline M & 1.5 & 1.5 & $X_{0} \quad(\mathrm{MPa})$ & 153 & 6.9 \\
\hline \multirow[t]{2}{*}{$\mathrm{N}$} & 1.27 & 1.29 & $C_{S D}$ & 4 & 3.9 \\
\hline & & & $C_{S L}$ & & 1.1 \\
\hline \multicolumn{3}{|c|}{ AFS model parameters } & $S_{\text {sat }}(\mathrm{MPa})$ & 387.2 & 246.7 \\
\hline$Y_{0}(\mathrm{MPa})$ & 308.3 & 122.2 & $n_{L}$ & 0 & 0 \\
\hline$\varepsilon_{0}$ & 0.00082 & 0.00308 & $n_{p}$ & 649 & 27.7 \\
\hline$n$ & 0.132 & 0.219 & $r$ & 0 & 1.9 \\
\hline$X_{s a t}(\mathrm{MPa})$ & 169.2 & 116.7 & $f$ & 0.862 & 0.415 \\
\hline$C_{X}$ & 15.8 & 1.45 & $C_{p}$ & 0.13 & 2.2 \\
\hline
\end{tabular}

Table 2. Damage parameters used in the simulations.

\begin{tabular}{ccccc}
\hline Parameters & $Y_{i}^{e}(\mathrm{MPa})$ & $S$ & $s$ & $\beta$ \\
\hline Values & 0 & 2 & 1 & 5 \\
\hline
\end{tabular}

Fig. 2 illustrates the effectiveness of the implementation of the damage model in the FE code Abaqus and its numerical validation. Since the damage model adopted in this work is not available in Abaqus, the validation is performed with respect to the isotropic-kinematic hardening model (without damage), which is available in Abaqus. First, the same hardening model is obtained from the Teodosiu model by setting several parameters to zero and by deactivating the damage. As shown in Fig. 2a, the reference curve is obtained even when extremely large time increments are used. Next, the same reference curve is recovered by giving limit values 
to the damage parameters, when the damage law is activated. Through these simple simulations, the numerical implementation of the model appeared to be accurate and effective.

Fig. 3 shows the stress-strain predictions of several monotonic and two-step sequential tests for the mild steel. The effects of strain-path changes are clearly captured by the Teodosiu model for both reverse and orthogonal tests, as reported by Haddadi et al. (2006). On the other hand, the coupling with the damage model introduces softening behavior effects that were not available in the previous versions of the model. Other two-stage strain-path loadings will be further simulated in $\S 5.2$ to analyze the effect of pre-strain on the FLD. Fig. 3b emphasizes the differences between the predictions of the two hardening models. As expected, the two models describe in a very different manner the transition zones after strain-path change; however, the predictions of the remaining of the two-stage sequential tests are remarkably similar up to very large strains. While this figure illustrates well the consistency of the two models, it is noteworthy that the predictions of the shear test up to shear strains equal to 3 cannot be considered realistic. In addition to the damage parameter identification, the choice of the Jaumann rate also becomes very questionable at such large strains. Indeed, although very popular in FE codes, this co-rotational description has been shown to exhibit some shortcomings when kinematic hardening is considered in the elastic-plastic constitutive modeling (Nagtegaal and De Jong, 1981; Lee et al., 1983). The main manifestation of this, which was first observed by Dienes (1979), is a spurious oscillatory shear stress response in simple shear test simulation when the strains become sufficiently large. This observation gave rise to extensive investigations in the literature; some of which suggesting the use of alternative objective stress rates, while a more consistent treatment of this undesirable oscillation, pioneered by Dafalias (1985), seems to be the inclusion of the plastic spin as a variable in the constitutive modeling. However, the evolution law of such a spin tensor is generally difficult to model; only micromechanical physically motivated models give an explicit formula for the plastic spin, whereas in most phenomenological models it is assumed to vanish. In all the subsequent applications, however, the stress-strain curves should not go beyond the strain localization point as discussed in Section 5. 


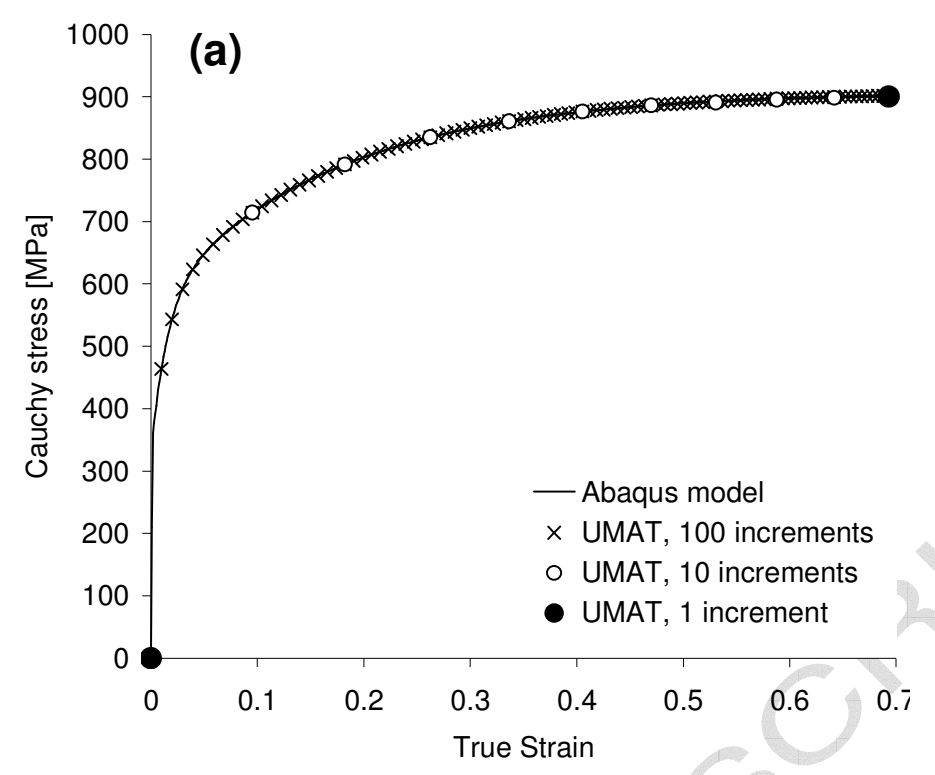

(b)

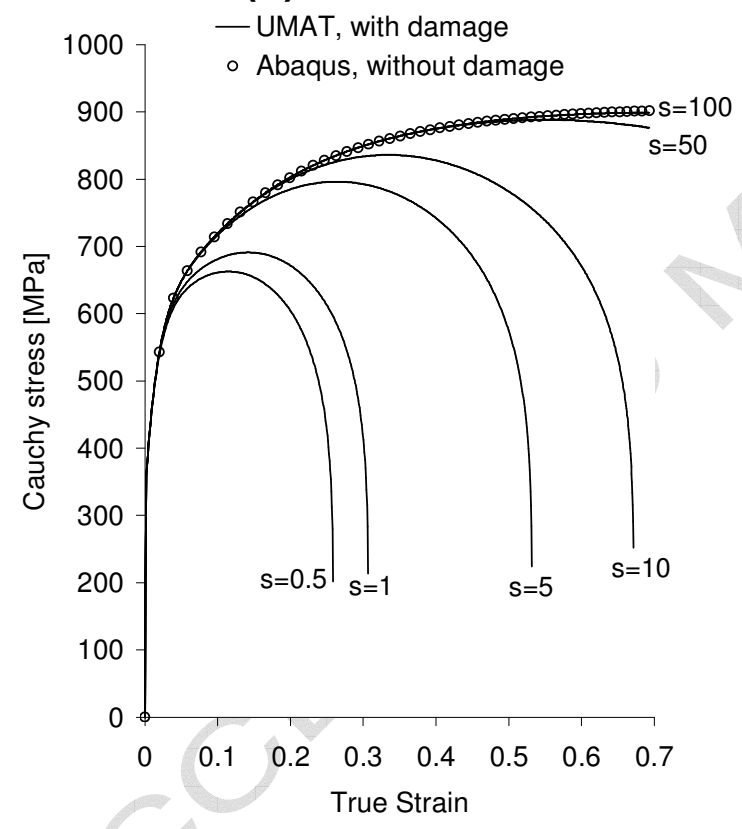

(c)

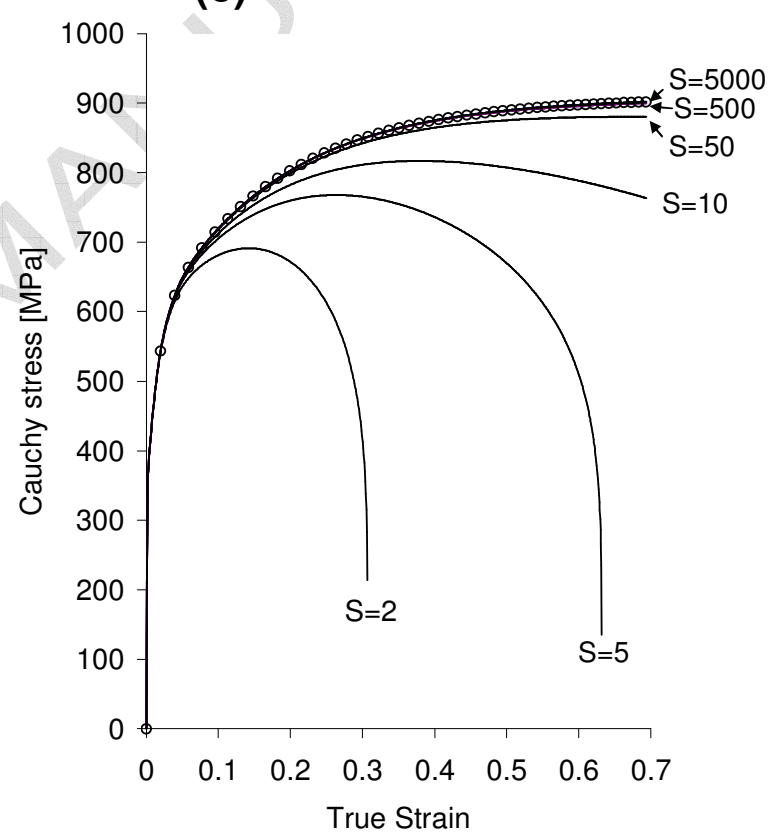

Fig. 2. Validation of the numerical implementation of the constitutive model; simulations of tensile tests for the DP material. (a) Validation of the elastic-plastic model with respect to the Abaqus built-in model. (b), (c) Effect of the damage parameters $s$ and $S$ on the stress-strain curve. 

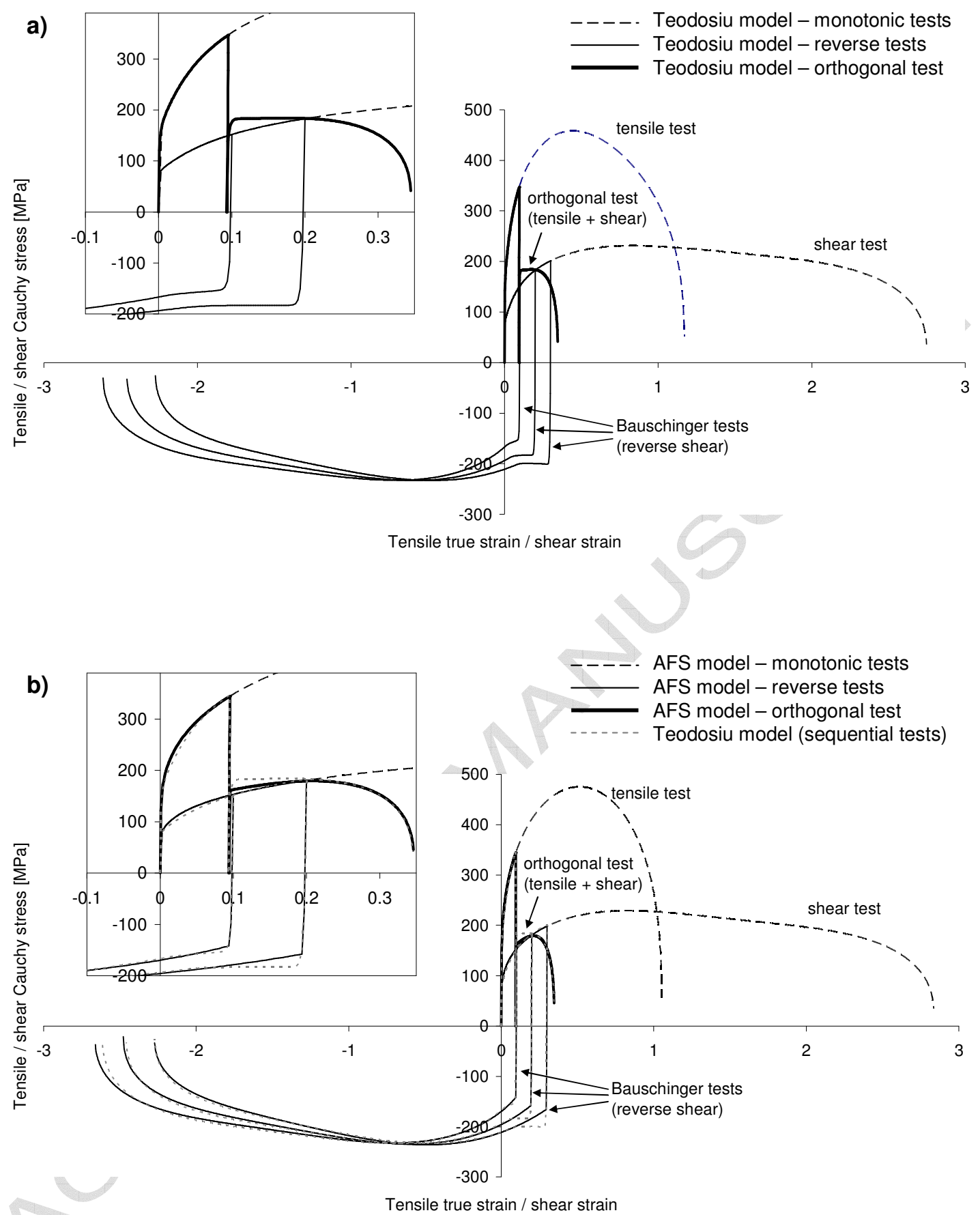

Fig. 3. Different loading path simulations for the mild steel using the damage model coupled to: a) the Teodosiu model, b) the Armstrong-Frederick-Swift (AFS) model. Monotonous tensile and shear tests (dashed lines), reverse shear tests (thin line), 10\% tensile test followed by a shear test (thick line). The $\left({ }^{\prime}\right)_{11}$ Cauchy stress / logarithmic strain components are represented for all situations but simple shear, when the shear Cauchy stress and shear engineering strain components $\sigma_{12}$ and $\gamma_{12}=2 \int D_{12} d t$ are plotted instead. The zones of moderate strains are enlarged to emphasize the transition zone predictions after strain-path changes. All the tests are performed along the rolling direction. 


\subsection{Forming Limit Diagram prediction}

As demonstrated by Rice (1976), the localization criterion considered here does not allow for the detection of strain localization in the case of an associative plasticity model with saturating stress-strain curves. This limitation is clearly illustrated in Fig. 4 (Top). In other terms, the introduction of a softening effect (by coupling the model with damage for instance) is required for the activation of the criterion, as shown in Fig. 4 (Bottom).

Fig. 5 illustrates the evolution of the localization criterion for different rheological tests as well as its respective predictions of limit strains. The strain state corresponding to the criterion activation is considered as the formability limit, and is plotted on the FLD. Fig. 6 shows the FLDs that correspond to various sets of damage parameters. As expected, delayed initiation of damage predicts higher formability limits. This figure clearly demonstrates the dramatic impact of the damage parameters on the formability prediction (see also Haddag et al. (2008)). 

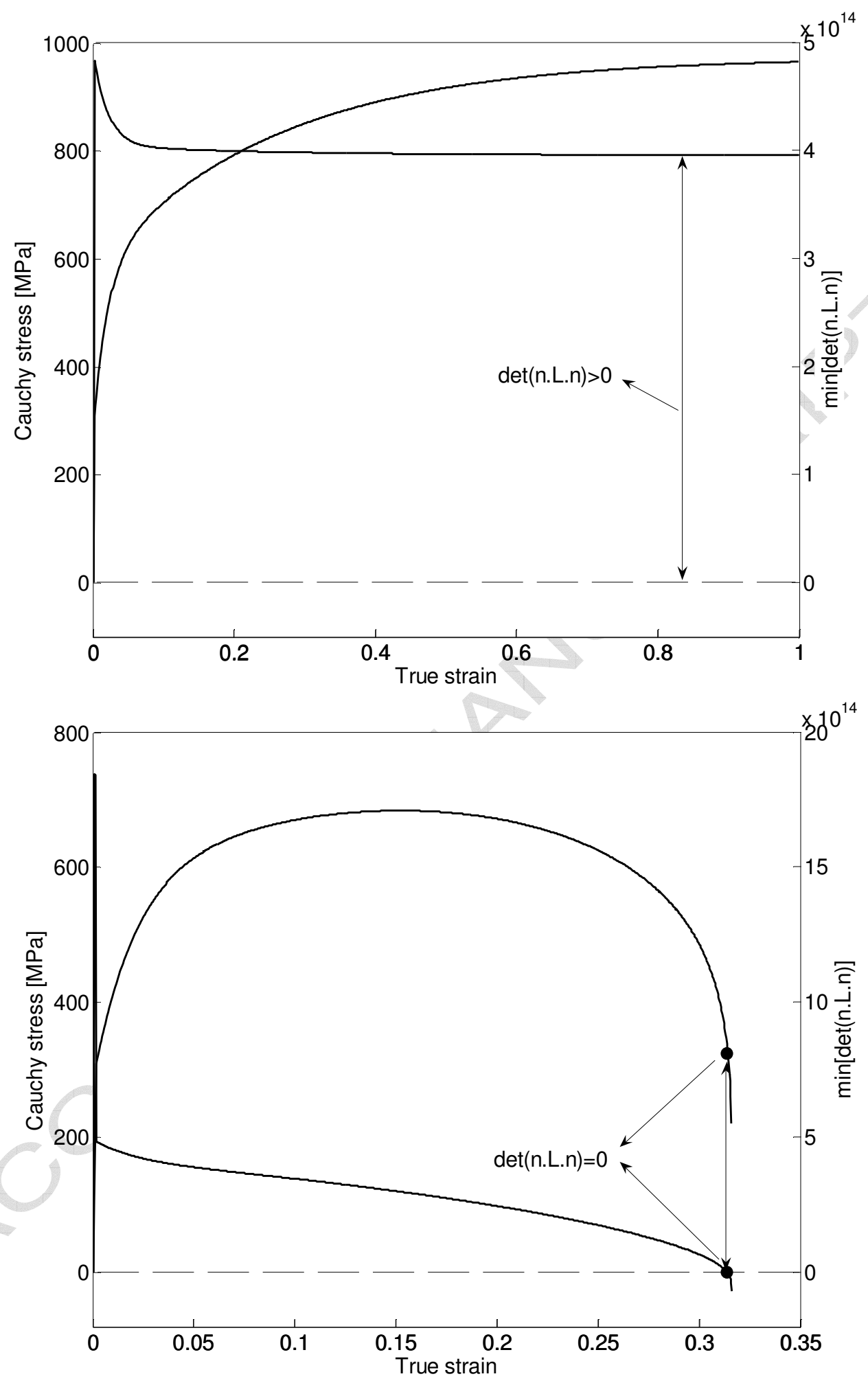

Fig. 4. Detection of strain localization by means of Rice's criterion in a uniaxial tensile test for the dual phase material without damage (Top) and with damage (Bottom). 


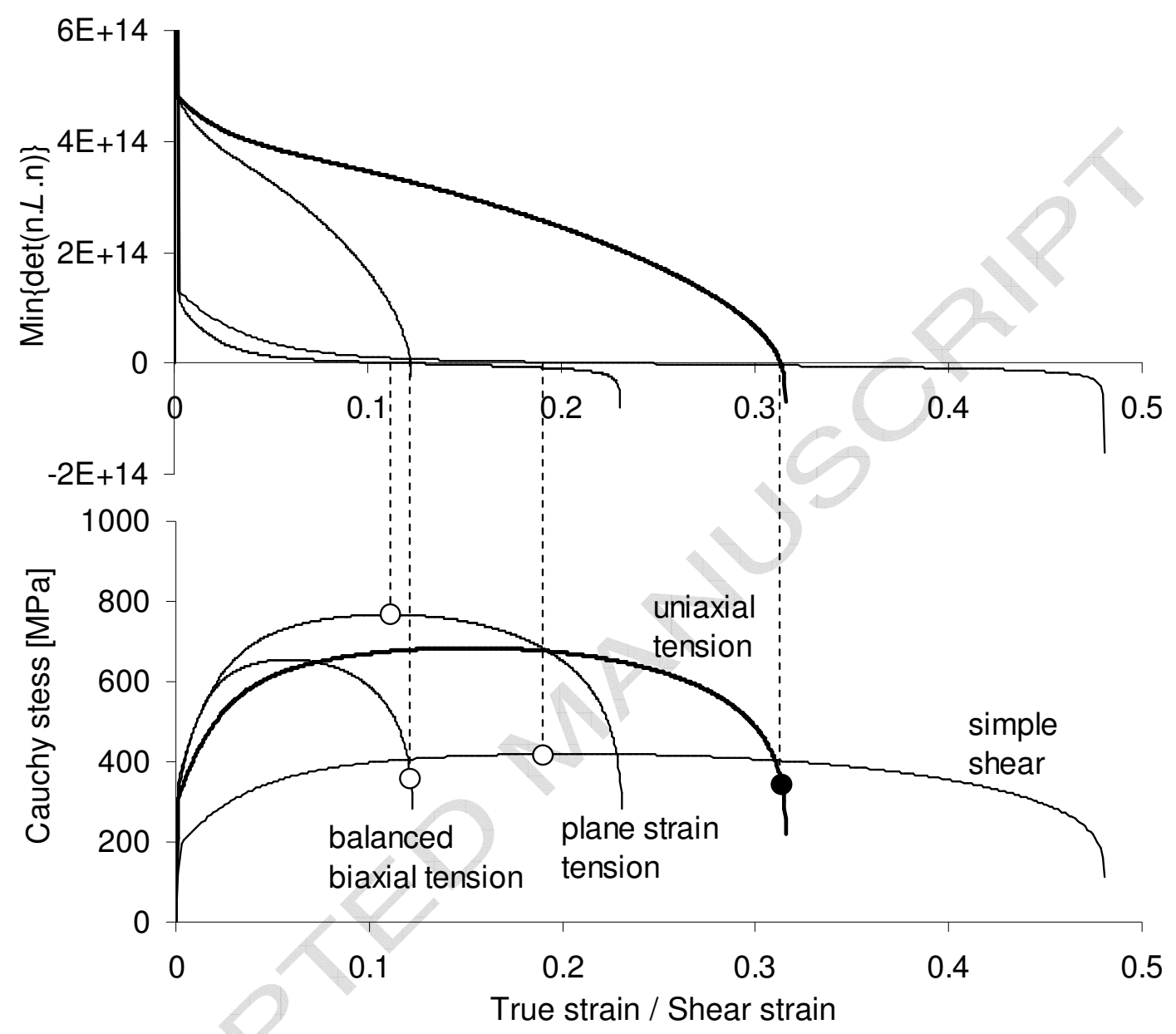

Fig. 5. Loading path simulations (bottom) and detection of strain localization by means of Rice's criterion (top) for the dual phase steel. 

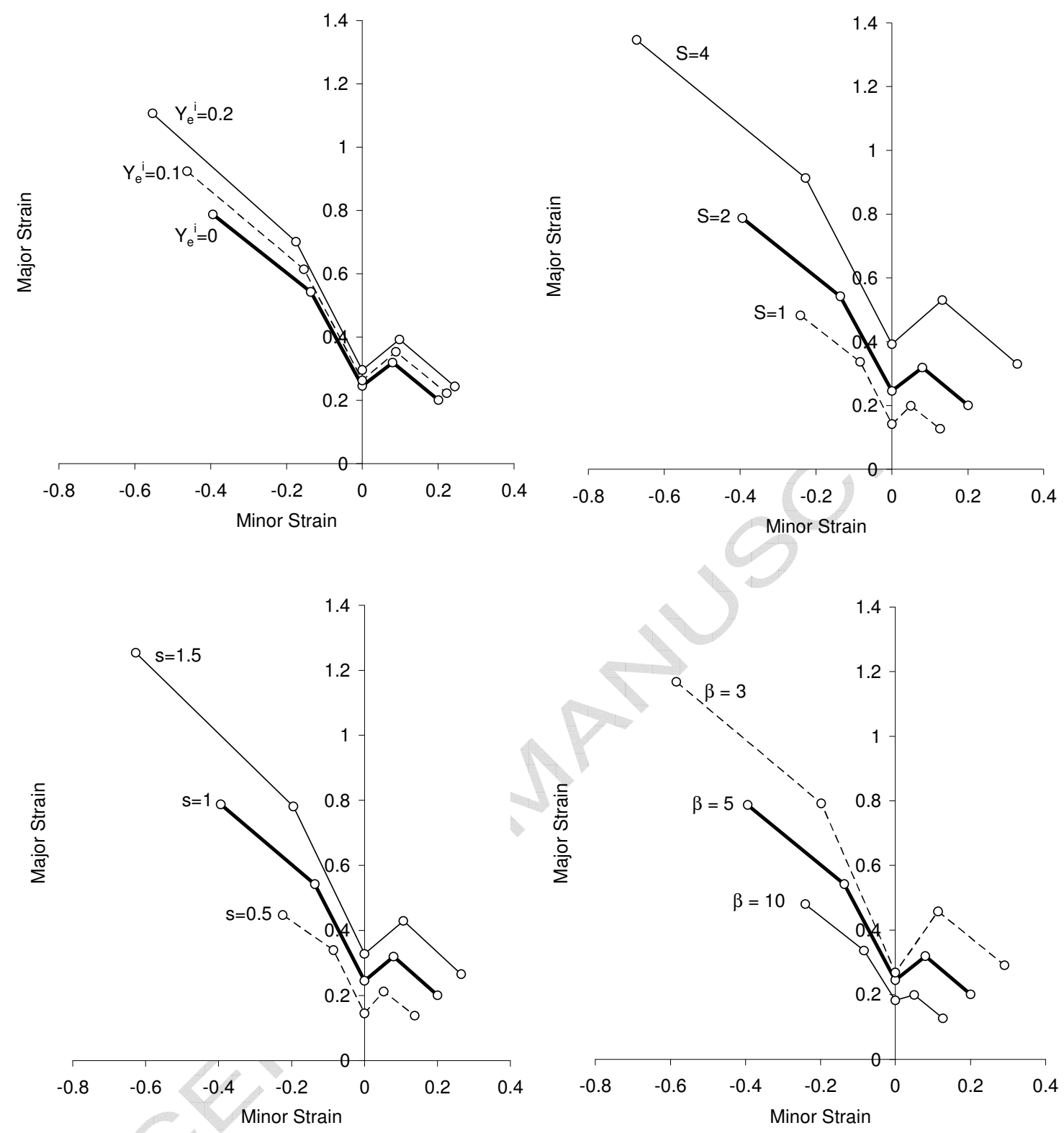

Fig. 6. FLDs for the mild steel predicted by means of the Rice criterion for different values of damage parameters. When not specified on the plots, the missing damage parameters take the values in Table 2 . The thick curve on all the plots corresponds to the damage parameters selected for all the subsequent simulations. 
In the remainder of the paper, the same damage model and the parameters from Table 2 are used with either the Teodosiu or Armstrong-Frederick-Swift (AFS) hardening model. In order to illustrate the consistency of this choice, the tensile stress-strain curves for the mild steel and the FLDs corresponding to both situations are plotted in Fig. 7. The respective predictions of the two models are slightly different. One may note that, in the range of moderate strains where the hardening parameters are usually identified (up to $30 \ldots 40 \%$ of tensile strain), the stress-strain curves almost coincide. Beyond the strain range that is typical for the identification of the hardening parameters, differences appear between the two predictions and, accordingly, the limit strains are somewhat different. As seen in Fig. 7a, the FLDs predicted by the two models are fairly close to each other, with the largest difference being observed for uniaxial tension. Finally, the effect of a pre-strain on the FLD is shown in Fig. 8. The well-known "translation" of the FLD to the left in the case of uniaxial tensile pre-strain is observed, as well as the translation of the FLD to the right in the case of balanced biaxial pre-strain. Thus, the classical tendencies observed in experiments are well reproduced by the localization criterion adopted in this work.

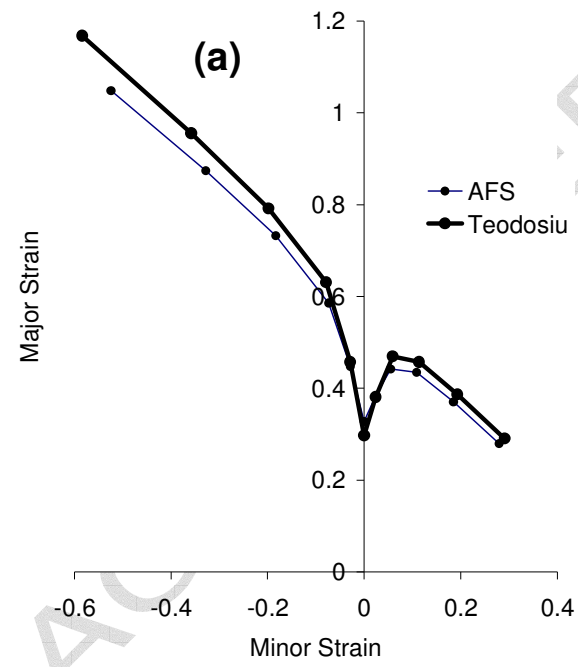

(b)

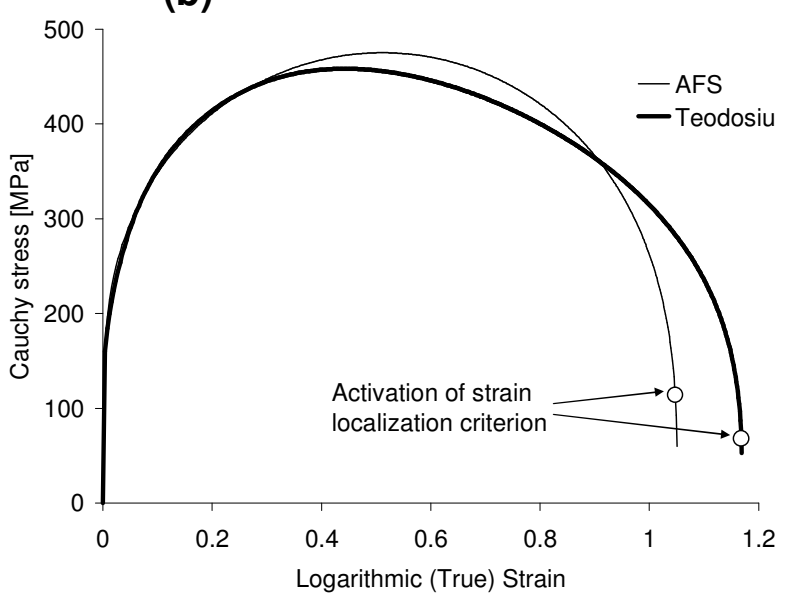

Fig. 7. Forming limit diagrams and tensile stress-strain curves for the mild steel predicted with the Teodosiu hardening model and the Armstrong-Frederick-Swift (AFS) model. The hardening parameters for both models are taken from (Haddadi et al., 2006), listed in Table 1, and the damage parameters are given in Table 2. 


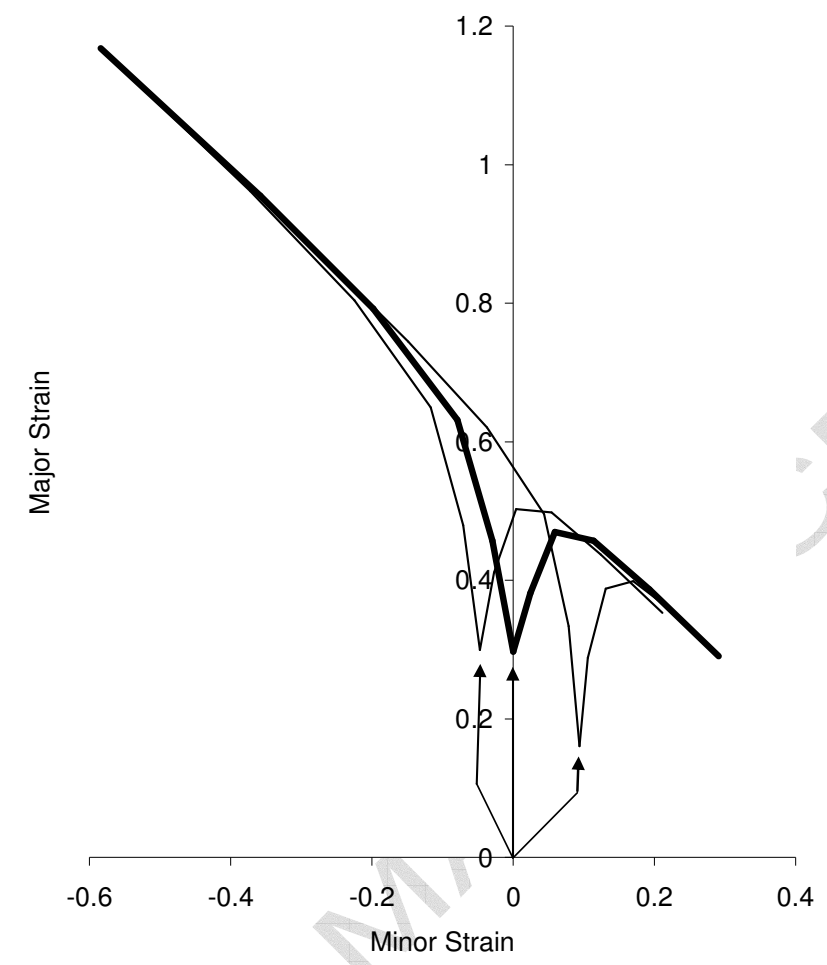

Fig. 8. Effect of $10 \%$ tensile and expansion pre-strains on the FLD predicted by means of Rice's criterion (mild steel, Teodosiu model).

\subsection{Orientation of the localization bands}

Rice's localization criterion also provides the orientation of the localization band. This orientation can be defined by two angles, as shown in Fig. 9: the angle $\theta_{1}$ gives the inclination of the band with respect to the rolling direction in the sheet plane (RD,TD), while the angle $\theta_{2}$ gives the inclination of the band with respect to the rolling direction in the thickness plane (RD,ND). For sheet materials, these two angles correspond to the in-plane orientation of the band, and a measure of its out-of-plane inclination. Most of the developments available in the literature assume a plane stress state and, moreover, do not take the out-of-plane inclination into account (e.g. Lemaitre et al., 2000). 


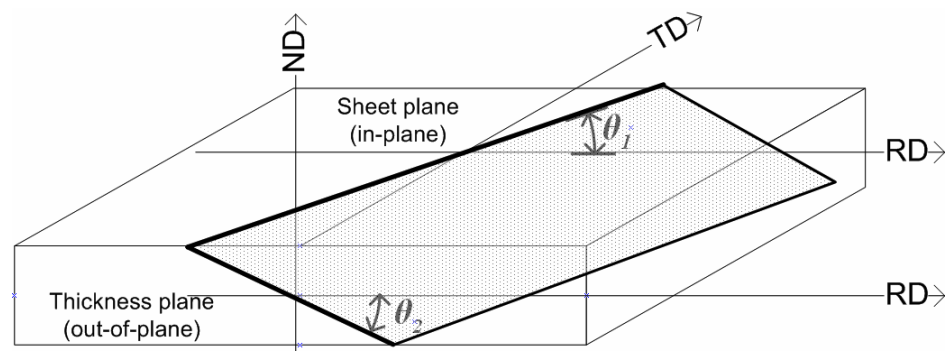

Fig. 9. In-plane and out-of-plane orientation of the localization band of the sheet.

The orientation of the localization band is reported in Fig. 10 for different loading modes. The values of the in-plane angle for simple shear and uniaxial tension correspond to the classically known experimental values as well as to those predicted with other models. For the plane strain tension, the result corresponds to the experimental observations, as well as to the prediction of the Marciniak-Kuczyński model (for the in-plane orientation). On the contrary, calculations of plane strain tension using the Rice model, when the normal to the localization band is forced to lay in the sheet plane, predict an in-plane angle of about $75^{\circ} \ldots 80^{\circ}$ (depending on the material model and parameters). Although the analysis is purely theoretical, the graphical representations clearly correspond to the experimental localization modes for the considered tests. The 3D analysis is not only useful for predicting the out-of-plane orientation of the band, but it is compulsory for a proper prediction of the in-plane orientation of the band and the corresponding limit strains. As shown in Fig. 10, the band is perpendicular to the sheet plane for simple shear and uniaxial tensile tests, while it is inclined at an angle of $45^{\circ}$ to the sheet plane for plane strain tension. In the case of the equibiaxial tensile test, there is no privileged in-plane orientation for the band. As a final result, the effect of pre-strain on the band orientation has also been investigated during sequential rheological tests. After $5 \%$ and $10 \%$ of pre-strain in uniaxial tension or equibiaxial tension are applied, the band orientations are practically unchanged during the subsequent monotonic tests. Thus, no effect of the pre- 
strain on the orientation of the localization band has been noticed. However, the limit strains are strongly influenced. This aspect is further investigated hereafter.
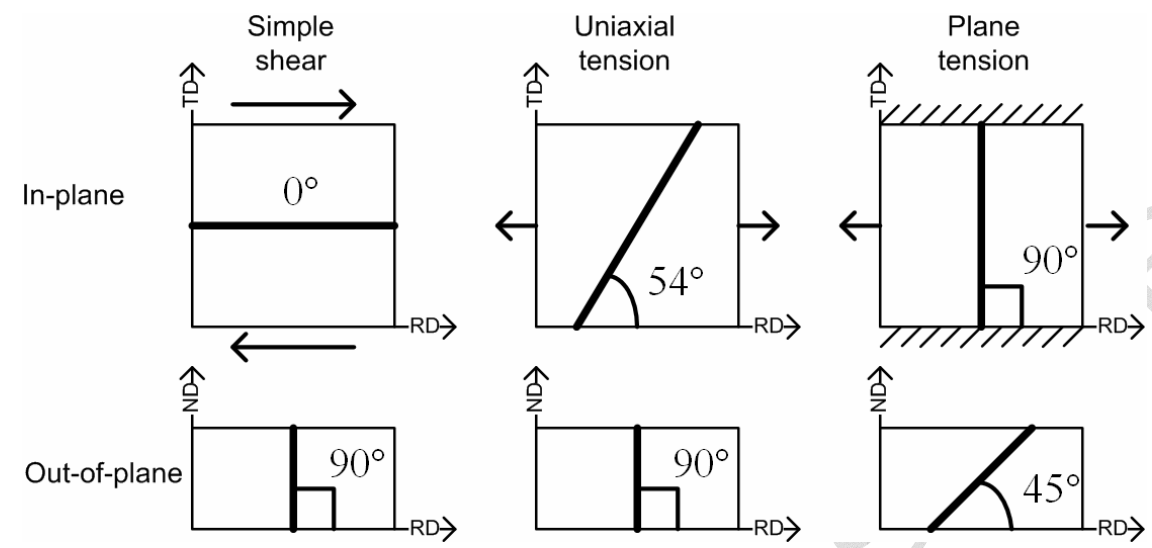

Fig. 10. Sketches of the in-plane and out-of-plane orientation of the localization band of the sheet.

\subsection{Influence of strain-path changes on the predicted FLDs}

The dramatic impact of the strain path on the forming limits is well known. The concept of stress-based FLD (the $\sigma$ FLD) initiated by Arrieux et al. (1985) sought to provide an alternative, strain-path independent way to address the sheet metal formability. However, the recent works of Yoshida and co-workers (Yoshida et al., 2007; Yoshida and Kuwabara, 2007; Yoshida and Suzuki, 2008) provide experimental evidence and theoretical explanations of the strain-path dependency of the $\sigma$ FLD for particular cases of strain-path changes and, more generally, when the constitutive behavior of the material is strain-path dependent. It has also been shown (Gotoh, 1985; Kuroda and Tvergaard, 2000b) that the details of the loading procedure during a strain-path change (with elastic unloading or not; with abrupt or continuous path change) strongly affect the results. Of equal importance, the constitutive model is known to affect the strain-path dependency of the FLD, at least when the Marciniak-Kuczyński model is used (Hiwatashi et al., 1998; Yoshida and Suzuki, 2008). Since Rice's criterion re- 
lies mainly on the constitutive tangent modulus to predict localization, its ability to capture the strain-path dependency on the FLD prediction is investigated here. Among several popular strain-path change possibilities (Nakazima et al., 1968), the combination of tensile or balanced biaxial pre-strains followed by plane strain tension has been shown to be insensitive to the details of the loading procedure adopted in the simulation (Kuroda and Tvergaard, 2000b). The mild steel is used for this investigation, as it exhibits complex strain-path-change transient phenomena, and the predictions of the Teodosiu model are compared to those of the AFS model (both of which are coupled with damage).

The results of this investigation are summarized in Fig. 11. When the second loading mode is plane strain tension, the formability of the sheet material is dramatically reduced. As soon as the amount of pre-strain reaches a certain level, the strain localization appears immediately after the strain-path change. It is clear from Fig. 11 that this critical pre-strain level is smaller for the Teodosiu model than for the AFS model, whatever the pre-strain is tensile or biaxial. In order to understand the origin of these differences, the stress-strain curves corresponding to the two-path tests used to determine the FLDs are shown in Fig. 12 for both models. The $(\cdot)_{11}$ stress and strain components are represented in these plots so that the strains at localization are the same as the major strain values for the corresponding points in Fig. 11. When tensile pre-strains are used (Fig. 12a and 12b), the results are very illustrative. With the increase of the pre-strain amount, the two models exhibit different characteristics. The classical AFS model always predicts almost the same plane strain tension curve, except that it is "translated" with the amount of tensile pre-strain. While the amount of pre-strain is increasing, the amount of subsequent strain prior to localization decreases and vanishes when the prestrain is $70 \%$ or larger. The Teodosiu model exhibits fairly different behavior characteristics. Larger pre-strains induce an increase in the stress level after strain-path change, together with a decrease of the slope of the stress-strain curve. As a consequence, the decrease of the amount of subsequent strain before localization is accelerated, and decreases more rapidly down to zero. The same conclusions can be observed in Fig. 12c and 12d for the second strain-path change, which involves a biaxial pre-strain followed by plane strain tension. These latter figures also show very clearly that the two models predict very different stress levels at localization. Indeed, the stress at localization is decreasing continuously for the AFS model as the pre-strain is increasing. However, for the Teodosiu model, the stress is increasing until the pre-strain reaches a value of $15 \%$. At this point, the subsequent strain (until localization) al- 
most vanishes and increasing the pre-strain leads to a decrease of the stress levels. This difference is due to the larger stresses and smaller hardening slopes predicted by the Teodosiu model after a strain-path change, in agreement with the experimental observations reported e.g. by Haddadi et al. (2006) for this material. These observations concerning the pathdependence of the stresses at localization agree very well with the recent conclusions obtained by Yoshida and Suzuki (2008) using the Marciniak-Kuczyński model.

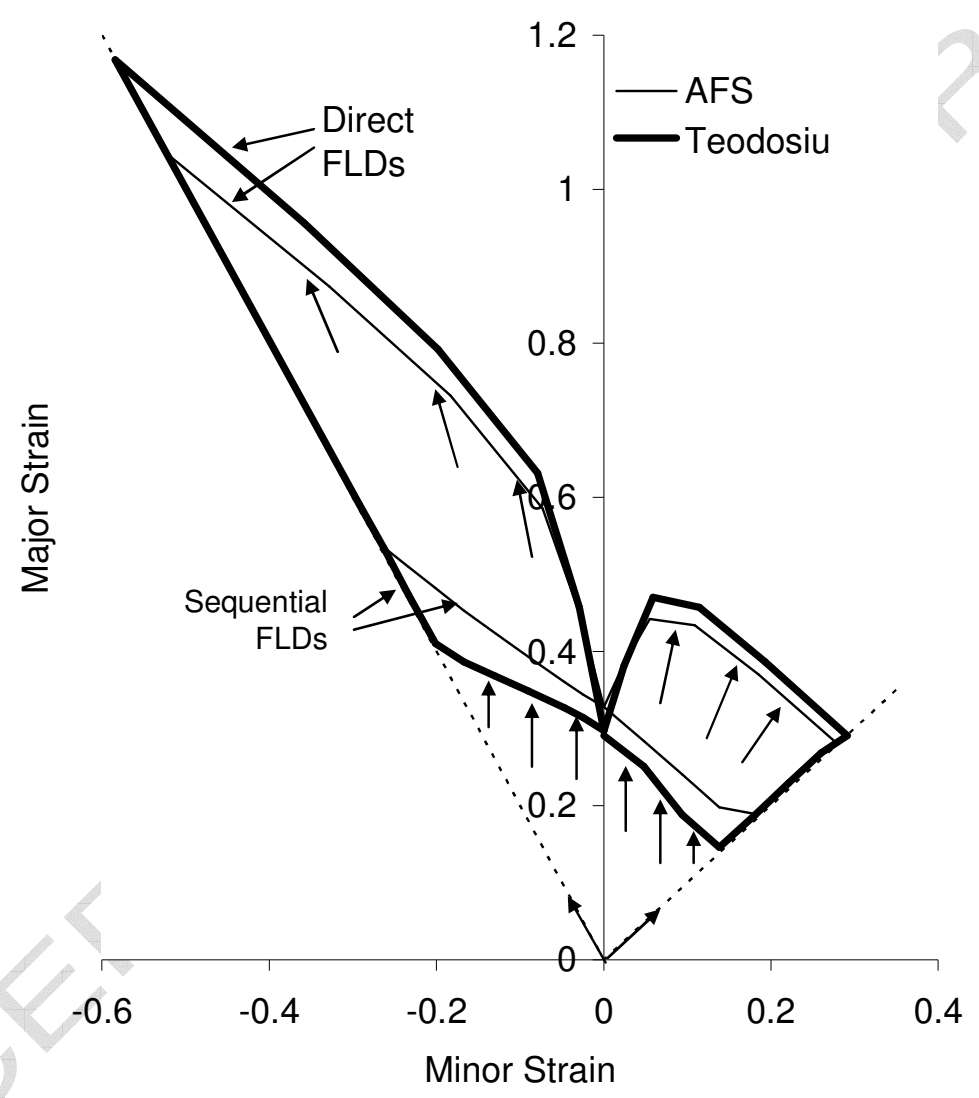

Fig. 11. Effect of strain-path change on the forming limit diagram of the mild steel: a comparison between the Teodosiu model and the Armstrong-Frederick-Swift (AFS) model. 

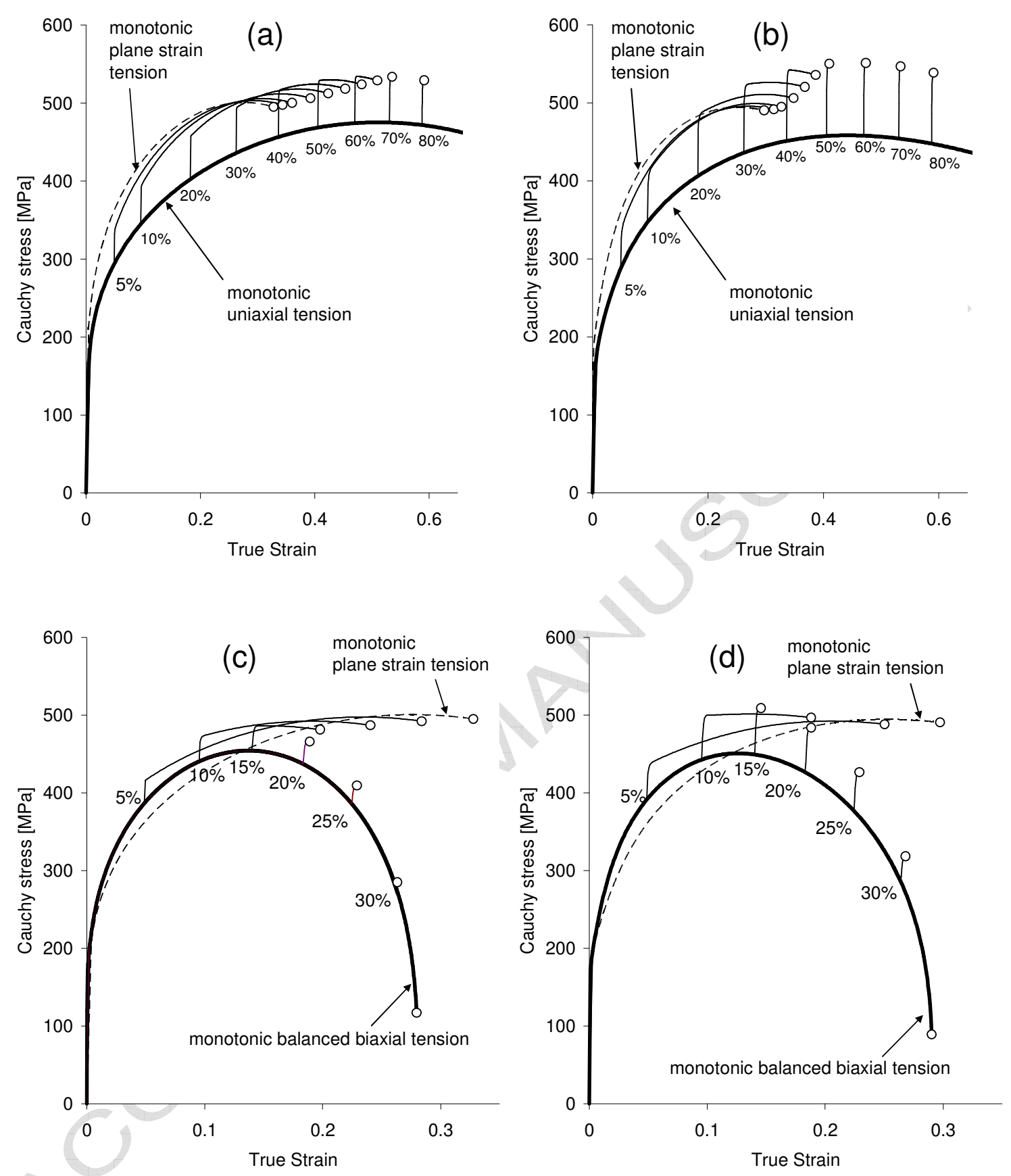

Fig. 12. Stress-strain curves for tensile loading followed by plane strain loading (a) with the AFS model and (b) with the Teodosiu model. (c) Stress-strain curves for balanced biaxial loading followed by plane strain loading with the AFS model and (d) with the Teodosiu model. Open circles indicate the points where the localization criterion has been reached for each simulation. The material used is mild steel. 
The two-step loading procedure used for this analysis did not include elastic unloading, as is clearly visible from the plots. The second loading path has been directly imposed at the end of the first path, and the stress point slides along the yield surface from the initial location to the subsequent one without unloading. This is consistent with Kuroda and Tvergaard (2000b), who showed the FLD to be insensitive to the loading procedure for this particular strain-path change. It is noteworthy that the transition zones after a strain-path change are correctly re-

produced here with the Teodosiu model, whether elastic unloading is simulated (Fig. 3) or not (Fig. 12), provided that the numerical implementation is accurate. This clarifies some discussions in the scientific literature about the need for a modified version of the Teodosiu model when the strain-path changes continuously.

\section{Conclusions}

An advanced anisotropic elastic-plastic model has been coupled to an isotropic damage model, and the bifurcation condition of Rice has been introduced as a localization criterion for this material model. The whole set of constitutive equations is formulated within the large deformation framework, since sheet metals undergo large strains during forming. In order to use the proposed approach as an effective prediction tool, a robust implementation of the constitutive model has been performed using an implicit integration scheme. The corresponding consistent tangent modulus has been derived, so that it can be used in an implicit finite element code.

For the strain localization analysis, an analytical tangent modulus has been developed within a general, fully three-dimensional framework, in order to predict the limit strains at localization, as well as the orientation of the localization band in the general 3D case, without the assumption of a plane stress state (as is commonly adopted in the literature). Also, the orientation of the planar band of localization has been sought in the whole space of possible orientations.

Monotonous and sequential two-step rheological tests have been simulated. As a first result, the proposed hardening-damage coupling allows for the description, simultaneously, of transient hardening due to strain-path changes and softening effects. These numerical simulations reproduce the experimental trends both in terms of limit strains and localization band 
orientation. In addition, the unconstrained, fully three-dimensional implementation of the localization criterion made out-of-plane orientations possible for the localization band, which represents an original result. The prediction of sequential FLDs after two-step loading strongly depends on the material model. The trends predicted with the Rice localization criterion used in this paper are confirmed by recent results from literature, obtained with the M-K model.

Consequently, the Rice criterion proves itself to be an effective alternative for the prediction of sheet metal formability. In order to further improve its accuracy, this modeling framework will be generalized to more complex (anisotropic) damage models and their numerical implementation in a fully implicit way. The model can be applied for the prediction of forming limits for arbitrary loading paths (e.g. with continuous strain-path changes), as well as strain localization predictions in finite element simulations of complex forming processes.

\section{References}

3DS report, 2001. Selection and identification of elastoplastic models for the materials used in the benchmarks. 18-Months Progress Report, Inter-regional IMS contract ' Digital Die Design Systems (3DS)'”, IMS 1999000051.

Abu Al-Rub, R.K., Voyiadjis, G.Z., 2003. On the coupling of anisotropic damage and plasticity models for ductile materials. Int. J. Solids Structures 40, 2611-2643.

Altenbach, H., Skrzypek, J.J., (Eds.) 1999. Creep and damage in materials and structures. Springer, Wien, New York (CISM Courses and Lectures No. 399).

Alves, J.L., 2003. Simulação numérica do processo de estampagem de chapas metálicas: Modelação mecânica e métodos numéricos. PhD Thesis, University of Minho, Portugal.

Aretz, H., 2007. Numerical analysis of diffuse and localized necking in orthotropic sheet metals. Int. J. Plasticity 23, 798-840.

Aretz, H., 2008. A simple isotropic-distortional hardening model and its application in elastic-plastic analysis of localized necking in orthotropic sheet metals. Int. J. Plasticity 24, $1457-1480$. 
Arrieux, R., Bedrin, C., Boivin, M., 1985. Determination of the strain-path influence on the forming limit diagrams from the limit stress curve. C.I.R.P. Annals 34/1, 205-208.

Barbier, G., Benallal, A., Cano, V., 1998. Relation théorique entre la méthode de perturbation linéaire et l'analyse de bifurcation pour la prédiction de la localisation des déformations. $\mathrm{C}$. R. Acad. Sci. Paris, 326, série II b, 153-158.

Benallal, A., 1998. Three-dimensional analysis of localized necking. In: de Borst, R., van der Giessen, E. (Eds.), Material instabilities in solids. John Wiley \& Sons Ltd, 254-268.

Benallal, A., Bigoni, D., 2004. Effects of temperature and thermo-mechanical couplings on material instabilities and strain localization of inelastic materials. J. Mech. Phys. Solids 52, Issue $3,725-753$.

Benallal, A., Billardon, R., Doghri, I., 1988. Integration algorithm and the corresponding consistent tangent operator for fully coupled elasto-plastic and damage equations. Communications in Applied Numerical Methods 4 (6), 731-740.

Benallal, A., Comi, C., 2000. On localization in saturated porous continua. C. R. Acad. Sci. Paris, 328, série II b, 847-853.

Bigoni, D., Hueckel, T., 1991. Uniqueness and localization-Part I: Associative and nonassociative elasto-plasticity. Int. J. Solids Structures 28 (2), 197-213.

Brunet, M., Arrieux, R., Boivin, M., 1985. Détermination par éléments finis en grandes déformations des courbes limites de formage en contraintes. Proc. Int. Symp. on Plastic instability, Paris, 227-238.

Brunet, M., Morestin, F., 2001. Experimental and analytical necking studies of anisotropic sheet metals. J. Mater. Proc. Technol. 112, 214-226.

Brünig, M., 2002. Numerical analysis and elastic-plastic deformation behavior of anisotropically damaged solids. Int. J. Plasticity 18, 1237-1270.

Brünig, M., 2003. An anisotropic ductile damage model based on irreversible thermodynamics. Int. J. Plasticity 19, 1679-1713. 
Brünig, M., Chyra, O., Albrecht, D., Driemeier, L., Alves, M., 2008. A ductile damage criterion at various stress triaxialities. Int. J. Plasticity 24, 1731-1755.

Butuc, M.C., Gracio, J.J., Barata da Rocha, A., 2006. An experimental and theoretical analysis on the application of stress-based forming limit criterion. Int. J. Mech. Sci. 48, 414-429.

Chaboche, J.L., 1986. Time independent constitutive theories for cyclic plasticity. Int. J. Plasticity $2(2), 149-188$.

Chaboche, J.L., 1999. Thermodynamically founded CDM models for creep and other conditions: CISM Courses and lectures $N^{\circ} 399$, International Centre for Mechanical Sciences. Creep and Damage in Materials and structures, 209-283.

Chaboche, J.L., 2008. A review of some plasticity and viscoplasticity constitutive theories. Int. J. Plasticity 24, 1642-1693.

Chow, C.L., Wang, J., 1987a. An anisotropic theory of elasticity for continuum damage mechanics. Int. J. Fracture 33, 3-16.

Chow, C.L., Wang, J., 1987b. An anisotropic theory of continuum damage mechanics for ductile fracture. Eng. Fracture Mech. 27, 547-558.

Chow, C.L., Yang, X.L., Chu, E., 2002. Prediction of forming limit diagram based on damage coupled kinematic-isotropic hardening model under non-proportional loading. J. Eng. Mater. Technol. 124, 259-265.

Cicekli, U., Voyiadjis, G.Z., Abu Al-Rub, R.K., 2007. A plasticity and anisotropic damage model for plain concrete. Int. J. Plasticity 23, 1874-1900.

Considère, A., 1885. Mémoire sur l'emploi du fer et de l'acier dans les constructions, Annales des Ponts et Chaussées, 9, 574.

Cordebois, J.P., Ladevèze, P., 1982. Détermination et étude des courbes limites de formage des tôles minces. Journées d'automne, Société Française de Métallurgie, 415.

Dafalias, Y.F., 1985. The plastic spin. J. Appl. Mech. 52, 865-871. 
Dienes, J.K., 1979. On the analysis of rotation and stress rate in deforming bodies. Acta Mechanica 32, 217-232.

Doghri, I., 2000. Mechanics of deformable solids: linear, nonlinear, analytical and computational aspects. Springer-Verlag, Berlin Heidelberg.

Doghri, I., Billardon, R., 1995. Investigation of localization due to damage in elasto-plastic materials. Mechanics of Materials 19, 129-149.

Dudzinski, D., Molinari, A., 1991. Perturbation analysis of thermo-visco-plastic instabilities in biaxial loading. Int. J. Solids Structures 27, 601-628.

Franz, G., Abed-Meraim, F., Ben Zineb, T., Lemoine, X., Berveiller, M., 2008. Strain localization analysis using a multiscale model. Comp. Mater. Sci. In press, doi:10.1016/j.commatsci.2008.05.033.

Franz, G., Abed-Meraim, F., Lorrain, J.P., Ben Zineb, T., Lemoine, X., Berveiller, M., 2009. Ellipticity loss analysis for tangent moduli deduced from a large strain elastic-plastic selfconsistent model. Int. J. Plasticity 25, 205-238.

Germain, P., Nguyen, Q.S., Suquet, P., 1983. Continuum thermodynamics. J. Appl. Mech. 50, 1010-1020.

Gotoh, M., 1985. A class of plastic constitutive equations with vertex effect - IV, Applications to prediction of forming limit strains of metal sheets under nonproportional loadings. Int. J. Solids Structures 21, 1149-1163.

Goya, M., Ito, K., 1990. An expression of elastic-plastic constitutive law incorporating stress increment dependence (initial material with Mises type plastic potential). JSME Int. J. Series 1. $33(1), 57$.

Gurson, A.-L., 1977. Continuum theory of ductile rupture by void nucleation and growth: Part I-Yield criteria and flow rules for porous ductile media. J. Eng. Mat. Tech. 99, 2-15. 
Haddadi, H., Bouvier, S., Banu, M., Maier, C., Teodosiu, C., 2006. Towards an accurate description of the anisotropic behaviour of sheet metals under large plastic deformations: Modelling, numerical analysis and identification. Int. J. Plasticity 22, 2226-2271.

Haddag, B., Abed-Meraim, F., Balan, T., 2006. Strain localization analysis using large deformation anisotropic elasto-plastic model coupled with damage. III European Conference on Computational Mechanics, C.A. Mota Soares et al. Eds., Lisbon, 1-14.

Haddag, B., Abed-Meraim, F., Balan, T., 2008. Strain localization and damage prediction during sheet metal forming, Int. J. Materials Forming, doi:10.1007/s12289-008-0362-z.

Haddag, B., Balan, T., Abed-Meraim, F., 2007. Investigation of advanced strain-path dependent material models for sheet metal forming simulations. Int. J. Plasticity 23, 951-979.

Hammi, Y., 2000. Simulation numérique de l'endommagement dans les procédés de mise en forme. PhD Thesis, Université de Technologie de Compiègne, France.

Hill, R., 1952. On discontinuous plastic states, with special reference to localized necking in thin sheets. J. Mech. Phys. Solids 1, 19-30.

Hill, R., 1958. A general theory of uniqueness and stability in elastic-plastic solids. J. Mech. Phys. Solids 6, 236-249.

Hiwatashi, S., Van Bael, A., Van Houtte, P., Teodosiu, C., 1998. Predictions of forming limit strains under strain-path changes: applications of an anisotropic model based on texture and dislocation structure. Int. J. Plasticity 14, 647-669.

Hora, P., Tong, L., Reissner, J., 1996. A prediction method for ductile sheet metal failure. Proceedings of the Numisheet'96, 252-256.

Hughes, T.J.R., Winget, J., 1980. Finite rotation effects in numerical integration of rate constitutive equations arising in large deformation analysis. Int. J. Num. Methods Engng. 15:12, $1862-1867$.

Hult, J., 1974. Creep in continua and structures. In: Zeman and Ziegler (Eds.), Topics in Applied Continuum Mechanics. Springer, New York, 137. 
Hutchinson, J.W., Neale, K.W., 1978. Sheet necking mechanics of sheet metal forming. Plenum Press, New York, 215-280.

Ito, K., Satoh, K., Goya, M., Yoshida, T., 2000. Prediction of limit strain in sheet metal forming processes by 3D analysis of localized necking. Int. J. Mech. Sci. 42, 2233-2248.

Kachanov, L.M., 1958. Time of the rupture process under creep conditions. Isv. Akad. Nauk. SSR. Otd Tekh. Nauk. 8, 26-31, in Russian.

Kachanov, L.M., 1986. Introduction to continuum damage mechanics. Martinus Nijhoff, Dordrecht.

Keeler, S.P., 1965. Determination of the forming limits in automotive stamping. Sheet Metal Industr. 42, 683-703.

Khelifa, K., 2004. Simulation numérique de l'endommagement en formage des structures minces. PhD Thesis, Université de Technologie de Troyes, France.

Krajcinovic, D., 1983. Constitutive equations for damaging materials. J. Appl. Mech. 50, $355-360$.

Kristensson, O., 2006. Numerically produced forming limit diagrams for metal sheets with voids considering micromechanical effects. Eur. J. Mech. A/Solids 25, 13-23.

Kuroda, M., Tvergaard, V., 2000a. Forming limit diagrams for anisotropic metal sheets with different yield criteria. Int. J. Solids Structures 37, 5037-5059.

Kuroda, M., Tvergaard, V., 2000b. Effect of strain path change on limits to ductility of anisotropic metal sheets. Int. J. Mech. Sci. 42, 867-887.

Kuroda, M., Tvergaard, V., 2004. Shear band development in anisotropic bent specimen. Eur. J. Mech. A/Solids 23, 811-821.

Laukonis, J.V., Gosh, A.K., 1978. Effects of strain-path changes on the formability of sheet metals. Metallurgical Transactions A 9, 1849-1856. 
Leckie, F.A., Hayhurst, D., 1974. Creep rupture of structures. Proc. R. Soc. Lond. A 340, $323-347$.

Lee, E.H., Mallet, R.L., Wertheimer, T.B., 1983. Stress analysis for anisotropic hardening in finite-deformation plasticity. J. Appl. Mech. 50, 554-560.

Lee, H., Peng, K., Wang, J., 1985. An anisotropic damage criterion for deformation instability and its application to forming limit analysis of metal plates. Eng. Fracture Mech. 21, 10311054.

Lemaitre, J., 1971. Sur la détermination des lois de comportement des matériaux élastoviscoplastiques. Thèse d'Etat, Université Paris XI, Orsay, France.

Lemaitre, J., 1984. How to use damage mechanics. Nuclear Eng. Design 80, 233-245.

Lemaitre, J., 1985. A continuous damage mechanics model for ductile fracture. J. Eng. Mat. Technol. 107, Issue 1, 83-89.

Lemaitre, J., 1992. A course on damage mechanics. Springer, Berlin.

Lemaitre, J., Chaboche, J.L., 1975. A nonlinear model of creep fatigue cumulation and interaction. In: Hult, J. (Ed.), Proc. IUTAM Symposium on Mechanics of Viscoelastic Media and Bodies, 291-301.

Lemaitre, J., Chaboche, J.L., 1985. Mécanique des matériaux solides. Editions Dunod, Paris. Lemaitre, J., Desmorat, R., Sauzay, M., 2000. Anisotropic damage law of evolution. Eur. J. Mech. A/Solids 19, 187-208.

Marciniak, Z., Kuczyński, K., 1967. Limit strains in the process of stretch forming sheet metal. Int. J. Mech. Sci. 9, 609-620.

Marquis, D., 1979. Modélisation et identification de l'écrouissage anisotrope des Métaux. Thèse de doctorat de l'Université Paris 6, France.

Murakami, S., 1983. Notion of continuum damage mechanics and its application to anisotropic creep damage theory. J. Eng. Mat. Tech. 105, 99-105. 
Murakami, S., 1988. Mechanical modeling of material damage. J. Appl. Mech. 55(2), 280286.

Nagtegaal, J.C., De Jong, J.E., 1981. Some aspects of nonisotropic work-hardening in finite strain plasticity. In: Lee, E.H., Mallet, R.L. (Eds.), Proc. of the Workshop on Plasticity of Metals at Finite Strain: Theory, Experiment and Computation. Division of Applied Mechanics. Stanford University, Stanford.

Nakazima, K., Kikuma, T., Hasuka, K., 1968. Study on the formability of steel sheets. Yawata Technical Report 264, 8517-8530.

Needleman, A., Tvergaard, V., 1983. Limits to formability in rate sensitive metal sheets. Proc. $4^{\text {th }}$ Int. Conf. Mechanical Behaviour of Materials, Stockholm, 51-65.

Pardoen, T., Doghri, I., Delannay, F., 1998. Experimental and numerical comparison of void growth models and void coalescence criteria for the prediction of ductile fracture in copper bars. Acta Mater. 46, 541-552.

Rabotnov, Y.N., 1969. Creep problems in structural members. North-Holland Publishing Company, Amsterdam.

Rice, J.R., 1976. The localization of plastic deformation. In: $14^{\text {th }}$ International Congress of Theoretical and Applied Mechanics, 207-220.

Rudnicki, J.W., Rice, J.R., 1975. Conditions for the localization of deformation in pressuresensitive dilatant materials. J. Mech. Phys. Solids 23 (6), 597-605.

Sánchez, P.J., Huespe, A.E., Oliver, J., 2008. On some topics for the numerical simulation of ductile fracture. Int. J. Plasticity 24, 1008-1038.

Signorelli, J.W., Bertinetti, M.A., Turner, P.A., 2008. Predictions of forming limit diagrams using a rate-dependent polycrystal self-consistent plasticity model. Int. J. Plasticity 25, 1-25.

Stoughton, T.B., 2000. A general forming limit criterion for sheet metal forming. Int. J. Mech. Sci. 42, 1-27. 
Swift, W., 1952. Plastic instability under plane stress, J. Mech. Phys. Solids 1, 1-18.

Teodosiu, C., 1997. Large Plastic Deformation of crystalline aggregates. CISM courses and lectures, Springer-Verlag, Austria.

Teodosiu, C., Hu, Z., 1995. Evolution of the intragranular microstructure at moderate and large strains: Modeling and computational significance. In: Simulation of Materials Processing: Theory, Methods and Applications. Numiform'95 Proceedings, 173-182.

Teodosiu, C., Hu, Z., 1998. Microstructure in the continuum modeling of plastic anisotropy. In: Proceedings of $19^{\text {th }}$ Riso International Symposium on Materials Science, Roskilde, 149168.

Thomason, P.F., 1990. Ductile fracture of metals. Pergamon press, Oxford.

Voyiadjis, G.Z., Kattan, P.I., 1992a. A plasticity-damage theory for large deformations of solids. Part I: Theoretical formulation. Int. J. Eng. Sci. 30, 1089-1108.

Voyiadjis, G.Z., Kattan, P.I., 1992b. Finite strain plasticity and damage in constitutive modeling of metals with spin tensors. App. Mech. Rev. 45, S95-S109.

Voyiadjis, G.Z., Kattan, P.I., 1999. Advances in damage mechanics: metals and metal matrix composites. Elsevier, Oxford, UK.

Wu, P.D., Lloyd, D.J., Jain, M., Neale K.W., Huang, Y., 2007. Effects of spatial grain orientation distribution and initial surface topography on sheet metal necking. Int. J. Plasticity 23, 1084-1104.

Wu, P.D., MacEwen, S.R., Lloyd, D.J., Jain, M., Tugcu, P., Neale, K.W., 2005. On prestraining and the evolution of material anisotropy in sheet metals. Int. J. Plasticity 21, 723739.

Yoshida, K., Kuwabara, T., 2007. Effect of strain hardening behavior on forming limit stresses of steel tube subjected to nonproportional loading paths. Int. J. Plasticity 23, 12601284. 
Yoshida, K., Kuwabara, T., Kuroda, M., 2007. Path-dependence of the forming limit stresses in a sheet metal. Int. J. Plasticity 23, 361-384.

Yoshida, K., Suzuki, N., 2008. Forming limit stresses predicted by phenomenological plasticity theories with anisotropic work-hardening behavior. Int. J. Plasticity 24, 118-139. 